Revue internationale P.M.E.

Économie et gestion de la petite et moyenne entreprise

\title{
Les facteurs de succès de la mise en place d'une communauté virtuelle dans les PME de services : le cas des petits et moyens cabinets d'experts-comptables
}

\section{Michel Vézina et Jacques Fortin}

Volume 15, numéro 3-4, 2002

URI : https://id.erudit.org/iderudit/1008813ar

DOI : https://doi.org/10.7202/1008813ar

Aller au sommaire du numéro

Éditeur(s)

Presses de l’Université du Québec

ISSN

0776-5436 (imprimé)

1918-9699 (numérique)

Découvrir la revue

Citer cet article

Vézina, M. \& Fortin, J. (2002). Les facteurs de succès de la mise en place d’une communauté virtuelle dans les PME de services : le cas des petits et moyens cabinets d'experts-comptables. Revue internationale P.M.E., 15(3-4), 63-99. https://doi.org/10.7202/1008813ar
Résumé de l'article

L'avènement des portails permettant la création de places d'affaires et de communautés virtuelles a profondément influencé le développement de l'Internet. Dans ce contexte, les PME ont la possibilité de faire valoir le poids de leur nombre en se regroupant sous forme d'association virtuelle de façon à profiter de ressources plus importantes et d'économies d'échelles substantielles.

Dans le domaine de l'expertise comptable, les petits et moyens cabinets d'experts-comptables (PMCCA) n'échappent pas à cette règle. Face à la mondialisation, les stratégies de gestion de la connaissance mises en œuvre par les grands cabinets d'experts-comptables et l'explosion de l'offre des services comptables informatisés sur le Web, les PMCCA n'ont d'autres choix que de se regrouper afin de demeurer concurrentiels.

Cet article présente la démarche de recherche qui a conduit à la création d'une communauté virtuelle pour les PMCCA. Celle-ci a débuté par l'élaboration d'un modèle théorique. Ce modèle s'est ensuite appuyé sur les résultats d'un processus de validation fait d'une enquête par questionnaire. Une fois validé, le modèle de départ a servi au développement d'un prototype opérationnel qui devait, par la suite servir à l'Ordre des comptables agréés du Québec à la conception d'une véritable communauté virtuelle des PMCCA. Celle-ci a d'abord porté le nom de Trousse CA et ne devait réunir que des comptables agréés du Québec. Le projet a depuis débordé les frontières du Québec et il est en voie d'être cédé à l'Institut canadien des comptables agréés (ICCA) qui pourrait en faire bénéficier l'ensemble des praticiens du Canada.

Les résultats de cette recherche-action tendent à démontrer que, si elle est prise en charge par une association professionnelle d'un minimum d'envergure, une communauté virtuelle peut être économiquement viable. Elle devra toutefois offrir à ses membres des produits et services à valeur ajoutée. Parmi la gamme de produits potentiels, il est intéressant de constater que les PMCCA accordent beaucoup plus de valeur aux produits d'information qu'aux produits qui facilitent les transactions en ligne.
Ce document est protégé par la loi sur le droit d'auteur. L’utilisation des services d'Érudit (y compris la reproduction) est assujettie à sa politique d'utilisation que vous pouvez consulter en ligne.

https://apropos.erudit.org/fr/usagers/politique-dutilisation/ 


\title{
Les facteurs de succès de la mise en place d'une communauté virtuelle dans les PME de services: le cas des petits et moyens cabinets d'experts-comptables ${ }^{1}$
}

\author{
Michel VÉZINA \\ Jacques FORTIN
}

École des Hautes Études commerciales

MOTS CLÉS

\author{
Portails - Communautés virtuelles \\ E-Commerce - Services comptables \\ Petits et moyens cabinets d'experts-comptables
}

\begin{abstract}
LES AUTEURS
MiChel VÉZINA, D.Sc, M.Sc., C.A., est professeur agrégé au service de l'enseignement des sciences comptables à l'École des Hautes Études commerciales de Montréal. II détient un doctorat en sciences de gestion de l'Université de Montpellier II en France. II est directeur et rédacteur en chef de la Revue internationale de gestion, membre du Groupe de recherche en système d'information (GReSI) ainsi que chercheur associé au Centre francophone en informatisation des organisations (CEFRIO). Adresse : École des Hautes Études commerciales, 3000, chemin de la Côte-Sainte-Catherine, Montréal, Québec, H3T 2A7. Courriel: michel.vezina@hec.ca

JACQUES FoRTIN, M.B.A., F.C.A., est professeur titulaire au service de l'enseignement des sciences comptables à l'École des Hautes Études commerciales de Montréal et associé universitaire de la firme Raymond Chabot Grant Thornton. Au cours de sa carrière, il a donné plusieurs conférences et publié plusieurs articles et ouvrages portant sur la mesure de performance financière et le contrôle de gestion. Adresse : École des Hautes Études commerciales, 3000, chemin de la Côte-Sainte-Catherine, Montréal, Québec, H3T 2A7. Courriel : jacques.fortin@hec.ca
\end{abstract}

1. Nous désirons remercier le Centre francophone en informatisation des organisations (CEFRIO) qui a subventionné ce projet de recherche ainsi que l'Ordre des comptables agréés du Québec (OCAQ) pour leur implication tout au long de ce projet. 


\title{
RÉSUMÉ
}

L'avènement des portails permettant la création de places d'affaires et de communautés virtuelles a profondément influencé le développement de l'Internet. Dans ce contexte, les PME ont la possibilité de faire valoir le poids de leur nombre en se regroupant sous forme d'association virtuelle de façon à profiter de ressources plus importantes et d'économies d'échelles substantielles.

Dans le domaine de l'expertise comptable, les petits et moyens cabinets d'experts-comptables (PMCCA) n'échappent pas à cette règle. Face à la mondialisation, les stratégies de gestion de la connaissance mises en œuvre par les grands cabinets d'experts-comptables et l'explosion de l'offre des services comptables informatisés sur le Web, les PMCCA n'ont d'autres choix que de se regrouper afin de demeurer concurrentiels.

Cet article présente la démarche de recherche qui a conduit à la création d'une communauté virtuelle pour les PMCCA. Celle-ci a débuté par l'élaboration d'un modèle théorique. Ce modèle s'est ensuite appuyé sur les résultats d'un processus de validation fait d'une enquête par questionnaire. Une fois validé, le modèle de départ a servi au développement d'un prototype opérationnel qui devait, par la suite servir à l'Ordre des comptables agréés du Québec à la conception d'une véritable communauté virtuelle des PMCCA. Celle-ci a d'abord porté le nom de Trousse CA et ne devait réunir que des comptables agréés du Québec. Le projet a depuis débordé les frontières du Québec et il est en voie d'être cédé à I'Institut canadien des comptables agréés (ICCA) qui pourrait en faire bénéficier l'ensemble des praticiens du Canada.

Les résultats de cette recherche-action tendent à démontrer que, si elle est prise en charge par une association professionnelle d'un minimum d'envergure, une communauté virtuelle peut être économiquement viable. Elle devra toutefois offrir à ses membres des produits et services à valeur ajoutée. Parmi la gamme de produits potentiels, il est intéressant de constater que les PMCCA accordent beaucoup plus de valeur aux produits d'information qu'aux produits qui facilitent les transactions en ligne.

\begin{abstract}
By making it possible to develop virtual market places and communities, the advent of portals has profoundly transformed the Internet. This has created a context that allows SMBs to take advantage of their strength in numbers by establishing virtual associations that offer them greater access to resources and substantial economies of scale.

Small and medium-size firms in the field of accounting (SMAFS) are no exception to this rule. Indeed, faced with the challenges represented by globalization, the knowledge management strategies developed by large accounting firms and the explosion of electronic accounting services on the Web, SMAFs have no other choice but to unite in order to remain competitive.

This article presents the research process that led to the development of a virtual community for SMAFs in Quebec. The process began with the formulation of a theoretical model. This model was then subjected to a validation process based
\end{abstract}


on the results of a questionnaire. Once validated, the initial model was used to develop an operational prototype that served as the basis for the creation by the Ordre des comptables agréés du Québec of an actual virtual community of SMAFs. Originally baptized the "Trousse CA », it was limited to chartered accountants in Quebec. The project has since been expanded beyond Quebec's borders and is in the process of being transferred to the Canadian Institute of Chartered Accountants (CICA), which could make its benefits available to all accountants in Canada.

The findings of this study suggest that, when overseen by a minimally large professional association, a virtual community can be economically feasible. It must, however, offer its members value-added products and services. Among the range of potential products, it is interesting to note that SMAFs place much more stock in information products than in products facilitating on-line transactions.

\section{RESUMEN}

El surgimiento de portales que permiten el desarrollo de sitios de intercambio comercial y de comunidades virtuales ha tenido una gran influencia en el desarrollo de la Internet. En tal contexto, se abre a las PyMEs la posibilidad de hacer valer el peso de su número al agruparse en una asociación virtual a fin de sacar provecho de mayores recursos y alcanzar importantes economías de escala.

En el campo de la actividad contable, las firmas de pequeña y mediana envergadura no constituyen la excepción a esta regla. Frente a la mundialización, las estrategias de gestión de los conocimientos desplegadas por las grandes firmas contables, y la explosión de la oferta de servicios contables informatizados a través de la Web, las firmas contables pequeñas y medianas no tienen otra alternativa que reagruparse para mantener su competitividad.

En este artículo se presenta la investigación que condujo al desarrollo de una comunidad virtual en el seno de las firmas contables pequeñas y medianas. El primer paso de tal investigación consistió en la elaboración de un modelo teórico que fue posteriormente avalado por los resultados de un proceso de validación realizado a través de una encuesta por cuestionario. Una vez validado, el modelo inicial sirvió de base para el diseño de un prototipo operativo que, a su vez, sería utilizado por el colegio profesional en cuestión, la Ordre des comptables agréés du Québec, para la creación de una comunidad virtual de firmas contables pequeñas y medianas. Esta comunidad, a la que se dio el nombre de Trousse CA, sólo debía originalmente agrupar a los profesionales contables matriculados de Quebec. Sin embargo, el proyecto traspasó las fronteras de esta provincia y actualmente se encuentra en vías de ser cedido al colegio profesional nacional, el Institut canadien des comptables agréés (ICCA), con lo que sus beneficios podrían alcanzar a todos los profesionales que ejercen en el territorio canadiense.

Los resultados de esta investigación tienden a demostrar que, si la iniciativa es iniciada por una asociación profesional con un mínimo de envergadura, una comunidad virtual puede resultar económicamente viable. Pero de todos modos, es preciso que la misma ofrezca a sus miembros productos y servicios con valor agregado. Entre la gama de productos posibles, resulta interesante observar que las firmas contables pequeñas y medianas acuerdan mayor valor a los productos de información que a aquellos que facilitan las transacciones en línea.

Revue internationale P.M.E., vol. 15, nos 3-4, 2002 


\section{ZUSAMMENFASSUNG}

Das Auftauchen der Webportale erlaubte die Entwicklung von Geschäftsgebieten und von virtuellen Gemeinschaften und hat die Entwicklung des Internets tiefgreifend verändert. In diesem Kontext haben die KMU die Möglichkeit, ihre gemeinsame Bedeutung neu zu bewerten, indem sie sich in Form von virtuellen Gemeinschaften gruppieren, und von den bedeutenden Ressourcen und von substantiellen ökonomischen Skalenerträgen profitieren.

Im Bereich der Buchführungsprüfung können die kleinen und mittleren Buchführungsbüros dieser Regel nicht entfliehen. Die kleinen und mittleren Buchführungsbüros sind konfrontiert mit der Globalisierung, mit den Führungsstrategien des entwickelten Wissens der grossen Buchprüfungsunternehmen und mit der Explosion von Buchhaltungsleistungen auf dem Web. Die KMU haben somit keine Wahl, sich zu gruppieren um ihre Wettbewerbsfähigkeit zu erhalten.

Dieser Artikel zeigt eine Forschungsanlage auf, die zur Entwicklung einer virtuellen Gemeinschaft für die kleinen und mittleren Buchführungsbüros geführt hat. Ausgangspunkt war die Ausarbeitung eines theoretischen Modells. Dieses Modell wurde nachfolgend abgestützt auf Resultate eines Validierungsprozesses, durchgeführt mit einer Untersuchung mittels Fragebögen. Einmal validiert, war das Ausgangsmodell nützlich, bei den anerkannten Buchführern in Québec einen operationellen Prototyp einer virtuellen Gemeinschaft zwischen kleinen und mittleren Buchführungsbüros zu entwickeln. Diese Gemeinschaft, mit dem Namen Trousse $C A$, sollte nur amtlich zugelassene Buchprüfer aus Québec umfassen. Das Projekt hat aber seitdem die Grenzen von Québec überschritten und man ist auf den Weg, das Konzept dem l'Institut canadien des comptables agréés (ICCA) zu übertragen. Mittels dem Institut könnten nachher alle Praktiker in Kanada von dieser virtuellen Gemeinschaft profitieren.

Die Resultate dieser Untersuchung versuchen aufzeigen, dass eine virtuelle Gemeinschaft ökonomisch lebensfähig ist, wenn ein Berufsverband mit einem Minimum an Weitblick sich um die Angelegenheit kümmert. Die Gemeinschaft muss jedoch den Mitgliedern Produkte und Dienstleistungen mit Mehrwert anbieten. Bei den möglichen Produkten ist es interessant festzustellen, dass die kleinen und mittleren Buchführungsbüros den Informationsprodukten mehr Bedeutung zumessen als Produkten, die On -line- Geschäfte ermöglichen.

\section{Introduction}

C'est au début du siècle dernier que les artisans de la comptabilité ont structuré leur métier autour d'ordres professionnels comptables. Sans retracer les origines de l'organisation professionnelle que l'on connaît maintenant, signalons simplement qu'au fil des ans, entre 1900 et 1950, trois ordres professionnels comptables ont vu le jour au Québec et que ces trois ordres constituent aujourd'hui la base de l'organisation professionnelle comptable au Québec : l'Ordre des comptables agréés du Québec, l'Ordre des comptables généraux licenciés du Québec et l'Ordre des comptables en management du Québec.

Revue internationale P.M.E., vol. 15, ns 3-4, 2002 
L'Ordre des comptables agréés réunit des professionnels qui exercent tous les métiers de la comptabilité et de la finance (contrôle de gestion, contrôle interne, production d'information financière, certification, analyses financières, financement, fiscalité, etc.), mais dont la particularité est de détenir l'exclusivité de la pratique de la certification des comptes des sociétés commerciales. En conséquence, c'est cet ordre professionnel qui, toutes proportions gardées, compte le plus grand nombre de membres qui travaillent en cabinet d'expertise comptable. L'Ordre des comptables agréés du Québec regroupe environ 16000 membres dont près de 6000 travaillent dans quelque 1500 cabinets privés. De ce nombre, près de 200 sont affiliés à ce qu'il est convenu d'appeler les cinq grands, 75 environ sont associés au réseau Raymond Chabot Grant Thornton (le plus grand des cabinets de moyenne dimension au Québec) et la moitié de ceux qui restent comptent deux associés et plus alors que l'autre moitié concerne les praticiens exerçant seul. Ces cabinets de comptables agréés représentent l'essentiel de la pratique comptable privée au Québec.

L'Ordre des comptables généraux licenciés réunit des professionnels qui, eux aussi, pratiquent à peu près tous les métiers de la comptabilité avec une certaine concentration cependant sur la production d'information financière et sur la gestion financière. Ils le font, toutefois, principalement au sein des organisations. L'Ordre des comptables en management accrédité rassemble des professionnels de la comptabilité dont le principal intérêt est le contrôle de la gestion au sein des entreprises.

C'est donc en conséquence de l'autonomie que leur procure l'exercice exclusif de la pratique de l'audit que les cabinets de services professionnels se sont principalement développés chez les comptables agréés. Le phénomène n'est d'ailleurs pas propre au Québec puisque, partout dans le monde, le marché des services professionnels en expertise comptable est très largement dominé par les cabinets dont la propriété est celle des professionnels de la certification. C'est principalement ce qui explique que, dans le contexte de cette recherche, le partenariat se soit établi avec l'Ordre des comptables agréés et que la population étudiée soit celle des cabinets de comptables agréés.

\section{Problématique}

\subsection{Technologies de l'information et comptabilité professionnelle, la genèse}

Jusqu'au tout début des années 1970, la certification accaparait la part la plus importante de l'activité des cabinets d'experts-comptables. Même si, à ce momentlà, l'informatique avait déjà fait son entrée chez les clients des experts-comptables, elle n'avait que très peu affecté les pratiques professionnelles. En effet, c'était l'époque des gros ordinateurs utilisés principalement pour tenir des livres et

Revue internationale P.M.E., vol. 15, nos 3-4, 2002 
accumuler des données. C'était aussi l'époque de la vérification autour de l'ordinateur. Ce fut enfin la période au cours de laquelle l'ordinateur au service du traitement de texte fit son entrée dans les cabinets.

Au début des années 1980, plusieurs grandes organisations, tant publiques que privées, ont entrepris de sélectionner leur vérificateur en procédant par voie de soumissions. Elles ont ainsi poussé à la baisse les honoraires de vérification et, par le fait même, ont diminué l'intérêt des cabinets pour ce type d'activité. Ce fut là, en quelque sorte, ce qui donna le coup d'envoi à une véritable diversification des services professionnels offerts par les cabinets d'experts-comptables. Tant les petits que les grands cabinets d'experts-comptables s'engagèrent dans le conseil en gestion, la fiscalité, le financement corporatif, la planification financière personnelle et le conseil en développement et en implantation de systèmes d'information (Olson, 1980; Hickok, 1982; Briloff, 1994).

Les années 1980 furent également celles au cours desquelles, surtout grâce à l'avènement de la micro-informatique, les outils informatiques ont fait leur apparition dans la trousse des experts-comptables. Plusieurs études ont toutefois démontré (Vézina, 1995; Lafortune et McNeil, 1993; LeGrand, 1988) que le recours à l'informatique pour l'expertise comptable, notamment la certification, se limitait à quatre applications, soit le traitement de texte, le chiffrier électronique et ses dérivés du type construction d'états financiers préprogrammés, l'extraction et l'analyse de données par progiciels et la construction de graphiques ou de rapports de toutes sortes. Ces mêmes études ont révélé que l'intensité du recours à ces outils s'est accrue au cours de ces années, mais qu'à la fin des années 1990 plusieurs professionnels de la comptabilité vivaient toujours en marge des nouvelles réalités informatiques. Enfin, toutes les études s'entendent pour reconnaître que l'informatique de l'époque a contribué à l'amélioration de la productivité des professionnels de la comptabilité et de la certification (Pentland, 1993; Lafortune et McNeil, 1993 ; Peat Marwick, 1987; Wolfe, Bain et McPheters1989).

Même si les outils disponibles à ce moment-là permettaient d'améliorer sensiblement l'efficacité du travail traditionnel, ceux-ci n'en ont pas, pour autant, transformé la nature des produits offerts non plus que la façon de faire les choses. Simplement, un vérificateur ou un conseiller plus informatisé était un vérificateur ou un conseiller plus efficace, mais il demeurait un vérificateur ou un conseiller traditionnel.

La décennie 1990 fut beaucoup plus bouleversante pour le milieu de la pratique professionnelle comptable. Elle a débuté par plusieurs fusions de sociétés ouvertes qui ont entraîné une baisse de la clientèle de vérification. Du même coup, ces fusions ont accru l'importance des risques et conduit les cabinets d'expertscomptables à se regrouper eux aussi. Qui plus est, la loi fédérale sur les sociétés 
commerciales canadiennes a supprimé l'obligation faite aux grandes sociétés fermées de faire vérifier leurs états financiers. Par conséquent, plus que jamais la diversification s'imposait.

Parallèlement, l'informatique se transformait pour devenir technologie de l'information et de la communication. Grâce à la technologie Internet, une masse considérable d'information devenait, dans tous les domaines et, bien entendu, dans ceux de l'information financière, soudainement disponible en temps réel. C'est cette disponibilité en temps réel d'informations de toutes sources qui a véritablement remis en question la pratique comptable traditionnelle. D'une part, elle aggravait la perte de valeur de l'information financière traditionnelle à caractère historique. D'autre part, elle permettait de repenser fondamentalement les processus de vérification de même que les produits de la vérification en même temps qu'elle ouvrait de nouveaux marchés aux professionnels de l'information que sont les experts-comptables.

Les choses ont alors évolué très vite dans les grands cabinets d'expertise comptable (les big five). En 1995, O'Gorman citait le cabinet d'experts-comptables KPMG qui considérait que l'automatisation de la certification, c'était d'être branché à des réseaux de communication locaux et globaux, accéder à des collègues partout dans le monde par courrier électronique, avoir accès à des informations sur la vérification, la comptabilité, les données d'une industrie à travers le réseau, produire des rapports de qualité professionnelle en utilisant les traitements de textes et les logiciels graphiques, avoir accès au système d'information de gestion du cabinet, disposer d'un ordinateur portatif. Cinq ans plus tard, ce que KPMG considérait comme une percée technologique en 1995 est devenu la norme des grands cabinets.

À cela se sont cependant ajoutés de très puissants logiciels de certification qui permettent de programmer le travail en fonction du secteur économique auquel appartient le client ainsi que de l'analyse des risques du client. S'est aussi ajoutée toute une industrie du courtage de l'information qui fonctionne grâce à la mise en réseaux mondiaux de spécialistes de toutes catégories et à la création d'espaces de connaissance dans lesquels les clients de cabinets d'experts-comptables sont à même de trouver de l'information de toute nature, filtrée et organisée en fonction des besoins de leurs propres modèles d'affaires. On est enfin à établir des communautés virtuelles de clients pour favoriser le partage de connaissances et de ressources entre eux.

On envisage aujourd'hui l'information financière continue disponible sur le Web ainsi que la vérification continue de cette information financière. On imagine également qu'aux rapports financiers classiques construits suivant les normes comptables nationales s'ajoutent des bases de données sur l'entreprise, certifiées par le vérificateur, à partir desquelles l'investisseur ou l'analyste réaliserait ses propres extractions et construirait l'information en fonction de ses besoins spécifiques.

Revue internationale P.M.E., vol. 15, nos 3-4, 2002 
(Elliot, 1996; AICPA, 1998; Melançon, 1998). La certification du non-financier (Brackney et Helms, 1996; Helms et Brackney, 1998) devrait, elle aussi, connaître un essor important. La certification des systèmes de mesure de performance, la certification des risques, les certifications ISO, l'audit environnemental et les garanties de sécurité des transactions en ligne sont autant d'exemples de nouveaux produits d'attestation. On travaille enfin au développement de cabinets-conseils virtuels qui permettraient à un client donné, situé à un endroit donné de la planète, de poser une question sur un sujet qui le préoccupe et d'obtenir sa réponse, à l'intérieur d'un délai très court, en provenance du meilleur spécialiste mondial du sujet en cause au cabinet.

De toute évidence, compte tenu des investissements colossaux en recherche et en développement que suppose le virage «technologies de l'information», on peut aisément imaginer que ce sont principalement les grands cabinets d'expertscomptables qui dominent le jeu et conditionnent les tendances.

\subsection{Un monde dominé par les grands cabinets}

Sur les sites des grands cabinets d'experts-comptables tels que Ernst and Young, PricewaterhouseCoopers, KPMG et feu Arthur Andersen ${ }^{2}$ on trouve, par exemple, les services de cyberconsultants qui, moyennant un forfait annuel, répondent, à l'intérieur de deux jours ouvrables à toutes les questions qu'un client peut vouloir poser à un professionnel du cabinet. Ces cyberconsultants alimentent en retour une banque de données faite de toutes les questions posées par les cyberclients et des réponses qui ont été apportées. Ces questions-réponses sont classées par sujet, par secteur d'activité et par taille d'entreprises et sont accessibles à tous les clients qui s'abonnent à ce forum. Les questions sont également analysées pour établir quelles sont les problématiques qui sont considérées d'intérêt et d'actualité pour les membres de la communauté des affaires. Cela fait, des textes sont rédigés et placés à la disposition des participants à la communauté virtuelle. Ces sites offrent également un outil, fait de questions à répondre en ligne, qui permet à l'abonné de comparer ses processus aux meilleures pratiques de l'industrie et ainsi de mesurer ses performances. Certains grands cabinets offrent un site de gestion de la connaissance destiné aux professionnels du cabinet. Ce site contient notamment des informations sur plusieurs domaines ou industries tels que télécommunications, énergie, finance et grossistes. Ces sites, quelquefois accessibles par les clients, peuvent aussi proposer des outils de gestion de portefeuille, d'étalonnage, de budgétisation, de gestion des ressources humaines, de vérification interne, de technologies de l'information, d'alliances stratégiques, d'impartition, de gestion des

2. Notre étude des sites des grands cabinets d'experts-comptables a été effectuée avant la disparition du cabinet Arthur Andersen.

Revue internationale P.M.E., vol. 15, ns 3-4, 2002 
risques et bien d'autres encore. Certains proposent une rubrique références qui regroupe les publications du cabinet, des liens vers les meilleurs sites d'affaires et des guides d'affaires internationaux. On y retrouve plusieurs centaines de liens classés par catégorie. Enfin, ces communautés virtuelles de grands cabinets fournissent systématiquement une description des services offerts par le cabinet et, souvent, des modules de gestion de la connaissance qui filtrent et trient l'information en fonction des besoins des professionnels du cabinet et de la clientèle, des conseillers virtuels et des communautés de clients virtuelles. Certains sites contiennent des informations très détaillées sur les services offerts par le cabinet partout dans le monde auxquelles s'ajoute une section publications qui contient diverses publications d'intérêt pour la clientèle, tels des guides d'application des nouvelles normes comptables.

\subsection{La place des PMCCA}

Dans cet univers comptable virtuel dominé par les grands, quelle place reste-t-il aux petits et moyens cabinets d'experts-comptables (PMCCA)? Au moment où nous nous posions cette question (début 1998), en dehors des cinq grands, on dénombrait environ 1500 cabinets de CA au Québec dont la moitié environ appartenaient à des praticiens exerçant seul et l'autre moitié comptaient au moins deux associés. Pour répondre à notre question, nous avons analysé le site Web de quelque 600 cabinets de deux associés et plus ainsi que celui de quelques cabinets sélectionnés au hasard de praticiens exerçant seul.

L'étude a démontré que, sur les 620 cabinets recherchés, seulement $30 \%$ offraient une adresse de courrier électronique où il était possible de les rejoindre et $7 \%$ possédaient un site Internet ${ }^{3}$. Au moment où cette portion de la recherche fut réalisée, les données provenant d'une étude du CEFRIO démontraient que $39 \%$ des entreprises montréalaises étaient présentes sur Internet. De toute évidence, le monde de la comptabilité ne contribuait que très faiblement à la société québécoise virtuelle.

De plus, sur les 43 sites de cabinets annoncés, $19 \%$ ne fonctionnaient pas, $12 \%$ étaient en construction et ne présentaient aucune information sur le cabinet, $19 \%$ ne donnaient que les noms et adresses du cabinet ainsi qu'une description sommaire des services offerts, $23 \%$ présentaient de l'information détaillée sur le cabinet, $14 \%$ avaient en plus des sections plus élaborées comme une revue de presse ou encore des possibilités de carrière et $5 \%$ offraient des services aux clients de type forums, outils interactifs ou documents à télécharger.

3. Deux engins de recherche ont été utilisés pour répérer les sites de cabinets : Altavista et le répertoire du site de l'Ordre des comptables agréés du Québec.

Revue internationale P.M.E., vol. 15, nos 3-4, 2002 
Une étude comparative a été menée en avril 2002 avec le même échantillon (Houle, Nzakimuena et Vézina, 2002). Les résultats de cette étude indiquent que même si le nombre de cabinets offrant une adresse électronique a doublé, le nombre de sites Web a, quant à lui, très peu évolué. En effet, en avril 2002, 59 \% des cabinets disposaient d'une adresse de courrier électronique où il était possible de les rejoindre alors que seulement $12 \%$ possédaient un site Internet, soit une augmentation d'à peine $5 \%$ en près de cinq ans. Toutefois, le degré de sophistication de ces sites s'est grandement accru. En effet, en avril 2002, ce sont $22 \%$ des cabinets sur le Web qui, en plus de présenter de l'information détaillée sur le cabinet, offrent des sections plus élaborées comportant une revue de presse ou encore des possibilités de carrière et $49 \%$ offrent des services aux clients de type forums, outils interactifs ou documents à télécharger. En conclusion, les cabinets présents sur le Web tendent à être de plus en plus sophistiqués, mais la plupart hésitent encore à investir dans la technologie Internet.

Lorsque l'on compare les résultats de l'analyse des sites des grands cabinets de CA avec les résultats de l'analyse des sites des PMCCA, on se rend vite compte que la petite pratique ne vit plus dans le même monde que la grande. L'écart d'intégration des technologies de l'information aux pratiques d'affaires des deux types de cabinets de CA est tel que l'on peut se demander si, dans vingt ans, il y aura encore de petites pratiques professionnelles comptables.

Plus encore, sur le terrain des services techniques de base, traditionnellement offerts par les petits praticiens (tenue de livres, gestion financière, conseils fiscaux, voire la certification), on s'aperçoit que de nouveaux concurrents, qui disposent de ressources importantes, s'apprêtent à entrer. Des institutions financières seront en mesure d'offrir les services de tenue de livres et de gestion de la trésorerie aux entreprises qui y effectueront toutes leurs transactions bancaires. Le ministère du Revenu travaille à développer des outils qui réduiront le recours aux intermédiaires entre eux et les contribuables. On voit même apparaître des franchises de la comptabilité qui offrent l'ensemble des services des petites pratiques et mettent à la disposition de leurs franchisés des instruments qui leur permettent de fonctionner à moindre coût et à efficacité accrue.

\subsection{Objectif et questions de recherche}

Pour survivre, dans le contexte actuel, il est essentiel que les PMCCA réagissent et réorientent leurs pratiques. Pour demeurer concurrentiels, ils doivent impérativement saisir les opportunités que leur offrent les technologies de l'information. Toutefois, la mesure du taux de branchement des PMCCA démontre clairement que des obstacles freinent leur virage technologique. Nous chercherons donc à établir quels sont ces obstacles pour ensuite proposer un concept qui inciterait ces organisations, pour leurs activités professionnelles, à recourir aux TIC et plus particulièrement à la technologie Web.

Revue internationale P.M.E., vol. 15, ns 3-4, 2002 
Par conséquent, l'objectif de cette recherche-action consistait à identifier l'application ou encore, le portefeuille d'applications dont l'attrait serait suffisamment grand pour inciter les PMCCA à se brancher et à prendre résolument le virage des technologies de l'information. Plus précisément, cette recherche cherchait à répondre aux questions suivantes :

1) Quels sont les besoins pour les petits et moyens cabinets d'expertscomptables (PMCCA) en ce qui concerne les applications informatiques?

2) Comment structurer l'offre d'applications informatiques destinée aux PMCCA afin qu'ils puissent demeurer compétitifs dans leur marché tout en tenant compte de leurs ressources limitées?

3) Quelles sont les applications les plus importantes pour les PMCCA ?

4) Existe-t-il des différences dans les besoins exprimés par les différentes catégories de PMCCA?

\section{Le développement d'un modèle théorique}

Deux étapes ont marqué le développement de notre modèle théorique :

1) l'identification des éléments à valeur ajoutée potentiels dont pourraient bénéficier les PMCCA en intégrant davantage les TI;

2) l'identification des composantes du modèle qui sera fait d'un ensemble d'applications potentiellement à valeur ajoutée.

\subsection{Première étape : L'identification des éléments à valeur ajoutée}

La façon la plus simple de bien saisir les potentialités des TI pour les PMCCA et, plus particulièrement, des technologies dérivées d'Internet, consistait à analyser les processus d'affaires des PMCCA, à décrire aux professionnels de ces cabinets les possibilités des technologies en cause et à réfléchir avec eux aux conséquences possibles d'un éventuel virage Internet.

Étant conscients de la faible utilisation de l'Internet par les PMCCA, nous estimions qu'il était risqué d'entreprendre l'identification des besoins des PMCCA sans avoir examiné les utilisations Internet possibles pour les experts-comptables. En outre, il nous paraissait évident que nos interlocuteurs éventuels éprouveraient autant de difficultés à identifier les services que la technologie en cause pourrait leur rendre qu'à imaginer les solutions technologiques appropriées.

Pour acquérir la connaissance préalable nécessaire, nous avons d'abord pris contact avec les cabinets qui étaient fortement engagés dans l'intégration des TI à leurs processus d'affaires, en l'occurrence, les cinq grands, dont nous avions déjà

Revue internationale P.M.E., vol. 15, $\mathrm{n}^{\text {os }} 3-4,2002$ 
analysé les sites. Ces entrevues ont permis de valider les conclusions que nous avions tirées de l'analyse des sites et accru notre compréhension des nouveaux produits et services issus de la combinaison TI et expertise comptable.

Par la suite, trois grands fournisseurs de services ont été rencontrés. Ainsi, nous avons pu confirmer les potentialités de la technologie Internet pour les PMCCA et réaliser dans quelle mesure les technologies en cause permettent maintenant à certaines entreprises, jadis tout à fait étrangères aux activités des expertscomptables, de s'accaparer d'une partie du marché des PMCCA. Par exemple, une grande institution financière s'apprêtait à lancer un produit Internet qui allait permettre aux PME de tenir leur comptabilité et d'effectuer la gestion de leur trésorerie en ligne sur le site de cette institution. Ce nouveau service menaçait directement les services de tenue de livre offerts par les PMCCA. En revanche, la même institution envisageait le développement de la confirmation bancaire en ligne qui pourrait grandement accélérer et faciliter le travail de vérification effectué par les experts-comptables.

Bien conscients que les outils Internet développés par les grands cabinets ne pouvaient pas s'appliquer directement aux PMCCA, nous avons tenté d'adapter les connaissances acquises lors des entrevues avec les cinq grands au contexte des PMCCA. Pour ce faire, nous avons rencontré des représentants d'un échantillon de PMCCA. Les principaux associés de cinq PMCCA situés à Montréal et en région et dont le nombre variait entre 2 et 12 nous ont accordé une entrevue. Nos entrevues, menées de façon semi-structurée et construites autour des fonctionnalités découvertes chez les grands, nous ont permis de faire un certain nombre de constats dont les principaux sont résumés ci-dessous :

- Il faut éviter de transposer les outils informatisés et les banques de données développées pour les grands cabinets aux petits cabinets sans les adapter aux besoins des PMCCA et à leur clientèle. Par exemple, il est évident que les conseils fiscaux en matière de prix de transfert ou encore les conseils portant sur la comptabilisation des opérations des filiales s'adressent généralement aux grandes entreprises. Il est aussi évident que les PMCCA ne disposent pas des ressources nécessaires pour constituer des bases de données des meilleures pratiques en contrôle de gestion et qu'on ne peut s'attendre d'eux qu'ils aient accès à un large bassin de spécialistes œuvrant dans différents domaines de la gestion.

- Les CA des PMCCA ont de la difficulté à juger de la valeur des sites de gestion de la connaissance tels qu'on les trouve chez les cinq grands. C'est surtout le cas lorsqu'on leur suggère de vendre l'accès de ces sites à leur clientèle. Ils comprennent mieux lorsqu'ils utilisent ces sites pour réduire leur propre temps d'accès à l'information dont ils ont besoin pour exercer leurs activités.

Revue internationale P.M.E., vol. 15, ns 3-4, 2002 
- Pour les petits cabinets, le coût a une incidence majeure sur les décisions d'achat. Par conséquent, tout nouvel outil ne sera acquis par les PMCCA que s'il a su démontrer un bon rapport qualité-prix. Comme le mentionnent Dyer (1997) ainsi que Amit et Zott (2000), l'utilisation du Web doit entraîner non seulement une réduction des coûts de recherche d'information et des coûts de transaction, mais également une réduction du coût des biens et services reçus.

- De plus, les PMCCA doivent être en mesure de pouvoir transférer, du moins partiellement, le coût des technologies à leurs clients. Il est également important de trouver le moyen d'offrir à leurs clients un service qui contribue à les fidéliser. Il s'agit en fait du concept de lock in présenté par Porter (2001) et Amit et Zott (2000).

- Les réticences des PMCCA sont fortement liées aux contraintes budgétaires qui leur sont imposées par leurs clients.

- Toutefois, dès qu'on illustre ce que pourrait être le contenu d'un site de gestion de la connaissance adapté aux besoins des PMCCA (cours à distance, bibliothèques fiscales, exemples de présentation d'information financière, banques d'occasions d'affaires ou consultation d'experts en ligne), l'intérêt augmente. Il a d'ailleurs été particulièrement élevé pour les bibliothèques fiscales. En effet, nos répondants comprennent assez bien à quoi pourrait leur être utile une base de données contenant les principales références fiscales. Dans ce cas, parce qu'ils connaissent le coût de ces instruments de travail, il est plus facile pour eux d'apprécier le retour sur investissement.

- Pour les professionnels des PMCCA, il est cependant impératif que l'accès aux référentiels qui pourraient être disponibles sur Internet soit très rapide.

- Il semble que l'attrait des outils de communication tels que les forums de discussion avec des professionnels d'autres cabinets soit inversement proportionnel à la taille du cabinet. Plus la taille du cabinet est grande, moins les professionnels qui y travaillent sentiraient le besoin d'échanger avec des gens de l'extérieur.

Ce sont donc les répertoires conviviaux regroupant des sites d'intérêt pour les CA des PMCCA auxquels ceux-ci pourraient avoir accès, comme les professionnels de grands cabinets ont accès aux sites de gestion de la connaissance de leurs cabinets, et auxquels ils pourraient aussi, éventuellement, donner accès à certains de leurs clients, qui sont apparus comme les principales composantes du modèle émergent.

Revue internationale P.M.E., vol. 15, nos 3-4, 2002 


\subsection{Deuxième étape : L'élaboration d'une solution et l'identification des composantes du modèle}

Au sortir du processus d'entrevues, nous avions acquis la conviction que ce ne serait que dans le cadre d'une communauté virtuelle comptable que les composantes du modèle émergent deviendraient disponibles à un coût raisonnable pour les PMCCA. C'est donc ce concept que nous avons entrepris de travailler. Pour Karp, Stone et Yoels (1977), une communauté est formée de trois composantes : 1) une interaction sociale soutenue, 2) un partage d'intérêts et de valeurs et 3) un espace géographique délimité. Lawrence (1995) précise qu'une communauté professionnelle regroupant par exemple les experts-comptables se caractérise par : 1) une interaction sociale soutenue, 2) des standards communs et 3) des règles de participation. Selon Rothaermel et Sugiyama (2001), une communauté virtuelle à la particularité d'être soutenue par des moyens de communication électroniques et de ne comporter aucune limite géographique ou temporelle.

Selon Hagel et Armstrong (1997), une communauté virtuelle regroupe tout d'abord des personnes ayant des intérêts ou des besoins communs, en second lieu, la communauté regroupe les ressources dont ces personnes ont besoin. Selon Lechner, Stanoevska-Slabeva et Hua Tan (2002), les communautés virtuelles représentent un facteur clé de succès de l'économie digitale. Selon Hagel et Armstrong (1997) et Shapiro et Varian (1999), huit conditions sont indispensables au succès d'une communauté virtuelle :

- Elle se concentre sur un centre d'intérêt particulier, par exemple, l'expertise comptable.

- Le pouvoir appartient aux membres.

- Le maître d'œuvre doit très bien connaître les besoins des membres et savoir utiliser ses connaissances au profit de la communauté virtuelle.

- Elle est capable de localiser, de filtrer et de communiquer aux membres des renseignements adaptés à leurs besoins.

- Elle permet d'intégrer le contenu informatif à un environnement propice à la communication et aux échanges d'information entre les membres. Les membres peuvent poser des questions aux auteurs afin de mieux comprendre les informations publiées. Ils peuvent en apprécier la crédibilité en discutant entre eux.

- Elle valorise les informations produites par les membres eux-mêmes.

- Elle ne donne pas l'exclusivité à un seul fournisseur. La communauté virtuelle cherche à regrouper le plus grand nombre de ressources de qualité possible en mettant en présence des fournisseurs qui se concurrencent.

Revue internationale P.M.E., vol. 15, ns 3-4, 2002 
Le maître d'œuvre de la communauté virtuelle privilégie les intérêts des membres plutôt que ceux des fournisseurs lorsqu'il négocie auprès de ces derniers.

- Elle a pour objectif d'être profitable aux membres en leur offrant des ressources de qualité et des environnements dans lesquels ils pourront accroître leur propre pouvoir.

La communauté virtuelle que nous envisagions allait permettre aux PMCCA de se doter d'outils de travail susceptibles de réduire l'écart qui s'était creusé entre eux et les gros joueurs de la profession, grâce au partage à plusieurs des coûts de développement de cette communauté. Plus encore, on pourrait même imaginer que les services offerts par cette communauté virtuelle pourraient non seulement accroître l'efficacité des PMCCA, mais encore leur permettre d'élargir la gamme de services offerts aux clients.

Toutefois, comme le mentionne Vaast (2001), la façon dont les membres des communautés virtuelles utilisent les technologies dépend du contexte, du type d'activités et des interactions qui s'y développent. Par conséquent, pour appliquer ce concept aux PMCCA, il est nécessaire de considérer un certain nombre d'éléments spécifiques à cette communauté. Les principaux sont énumérés ci-dessous :

- Il existe un grand nombre de PMCCA;

- La compétition entre les PMCCA est très forte et, par surcroît, on assiste à un effritement du marché traditionnel des PMCCA au profit de nouveaux intervenants ;

- Malgré les ressources limitées dont ils disposent et l'intérêt qu'ils ont pour partager la connaissance et les expertises, les échanges entre les PMCCA sont assez rares ;

- Les PMCCA, comme l'ensemble des PME québécoises (Ouellet et Trudeau, 2001), sont peu outillés en termes de TIC;

- Les experts-comptables œuvrant au sein des PMCCA sont submergés d'information et la gestion de l'information leur pose de sérieux problèmes ;

- Les ressources humaines et financières des PMCCA sont limitées.

Ces constatations nous ont conduits à relever un certain nombre de besoins qui nous ont guidés dans le processus d'identification d'une solution appropriée. Parmi les éléments importants, notons :

- La nécessité d'offrir de l'information et des applications à un coût abordable ;

- L'intérêt d'un guichet unique pour répondre à tous les besoins ;

Revue internationale P.M.E., vol. 15, nos 3-4, 2002 
- La nécessité d'échanges électroniques sécuritaires;

- L'amélioration du réseau d'échange avec les autres experts-comptables ;

- La nécessité d'améliorer la gestion interne des données;

- L’importance de faciliter la gestion de la connaissance.

La solution appropriée devrait permettre d'ajouter de la valeur aux services actuels, de rendre de nouveaux services, d'établir des alliances et de partager des ressources. L'objectif qui apparaissait le plus évident consistait à regrouper et à favoriser les échanges entre PMCCA afin de créer la synergie qui leur permettrait de mieux faire face à la compétition. Selon Hagel et Armstrong (1997) et Jo Kim (2000), une communauté virtuelle doit mettre l'accent sur un domaine d'intérêt précis, offrir la possibilité d'intégrer la connaissance et les communications, s'enrichir par le biais de la contribution au contenu par les membres, faciliter l'accès à plusieurs fournisseurs qui se concurrencent et adopter une orientation commerciale. De plus, comme le mentionnent Hagel et Armstrong (1997), les communautés virtuelles offrent aux entreprises une chance de mieux connaître et ainsi de mieux servir leurs clients. Elle convient donc tout à fait à ce qui nous a semblé être souhaité par les PMCCA.

Toutefois, comme le signalent Hagel et Armstrong (1995), il importe de bien définir les composantes de la communauté virtuelle en fonction des besoins des utilisateurs. L'étape suivante consistait donc à établir, à partir des informations issues de l'analyse des sites Web des communautés virtuelles existantes dans d'autres domaines, de la revue de la littérature et des entrevues qui avaient précédé, les composantes pertinentes de la communauté virtuelle. Treize modules furent retenus pour concevoir le premier prototype qui fut baptisé @BACUS ; chacun d'eux est sommairement décrit ci-dessous.

1. Documentation officielle : Ensemble de textes publiés par des autorités de réglementation, formulaires, outils de vérification et guides d'application pratique.

2. Bases de connaissances et d'expertise : Bases de données adaptées aux besoins des très petites entreprises et des PME. On y retrouverait des exemples de notes aux états financiers, des ratios, des meilleures pratiques connues, des lacunes de contrôle interne, etc.

3. Cybermanuel : Manuel de référence de l'Institut canadien des comptables agréés (ICCA) en comptabilité et en vérification annoté et référencé électroniquement à des bases de données financières, juridiques, à des sites Web, etc. 
4. Service de recherche en ligne: Module par lequel les membres pourraient obtenir un avis écrit au sujet d'un problème de comptabilité, de vérification ou de fiscalité.

5. Formation virtuelle: Module diffusant des cours par Internet.

6. Applications transactionnelles : Module qui offrirait des applications innovatrices. Ces applications comprennent le courriel de confirmations bancaires, la sauvegarde de fichiers sur un serveur externe, l'utilisation d'un logiciel de vérification conservé sur le serveur de la communauté virtuelle.

7. Certificats numériques : Module qui offrirait des certificats numériques qui permettent de signer électroniquement des documents et de les crypter.

8. Boutique virtuelle : Boutique où l'on trouverait tous les types de logiciels pouvant intéresser les PMCCA de même que des ordinateurs. La boutique offrirait des rabais à ses membres, des évaluations indépendantes et un babillard sur lequel des commentaires pourraient être affichés.

9. Site Web : Module à partir duquel les PMCCA pourraient construire eux-mêmes leur site Web en quelques minutes. Le site Web est hébergé ou lié à la communauté virtuelle.

10. Webdiffusion (push) de nouvelles : Service personnalisé de nouvelles à caractère économique et professionnel.

11. Échanges entre CyberCA : Forums, cyberbavardage (chat) et babillards électroniques.

12. Répertoires virtuels : Bottins de bonnes adresses, mis à jour régulièrement avec moteur de recherche intégré.

13. Marché virtuel : Module «Fusions et acquisitions » qui permettrait de rapprocher acheteurs et vendeurs d'entreprises. Ce module offrirait aussi un Centre d'emploi virtuel.

\section{Méthodologie}

\subsection{La population visée}

L'évaluation des besoins des PMCCA a été basée à la fois sur l'examen des produits offerts par les grands cabinets et sur les préoccupations exprimées par les associés des PMCCA rencontrés dans le cadre d'entrevues semi-structurées. Cette démarche, comme le relèvent Lefebvre et Lefebvre (2001), présente l'avantage d'assurer une meilleure validité interne que les enquêtes par questionnaires tout en permettant d'obtenir plus de renseignements, et cela, à partir d'un échantillon plus petit.

Revue internationale P.M.E., vol. 15, nos 3-4, 2002 
Afin d'obtenir, comme le proposent Lefebvre et Lefebvre (2001), une meilleure validité externe tout en s'assurant que les modules proposés conviennent aux PMCCA et que les besoins et opinions émises dans le cadre du processus d'entrevue sont représentatives de l'opinion de l'ensemble des PMCCA, il s'est révélé nécessaire d'effectuer une enquête par questionnaires auprès d'un échantillon plus large de PMCCA.

L'enquête vise les PMCCA établis au Québec à la fin du printemps 1999. Ces PMCCA excluent les «Cinq Grands » de même que le cabinet québécois Raymond, Chabot, Grant, Thornton, cabinet comptant plus de 1000 employés. Ils englobent donc les praticiens exerçant seul, qu'ils soient maîtres de stage ou non, et les autres cabinets comptant plus d'un associé. La liste exhaustive et à jour des PMCCA a été fournie par l'Ordre des comptables agréés du Québec. Cette liste est mise à jour annuellement. Au moment de l'enquête, cette population comptait environ 1500 PMCCA.

\subsection{L'échantillon}

Au total, 1000 questionnaires ont été postés. L'échantillon comptait 592 praticiens exerçant seul (soit $51 \%$ de la population totale des praticiens exerçant seul) et 408 PMCCA comptant plus d'un associé (soit $100 \%$ de la population totale des autres cabinets). En résumé, près de $64 \%$ de la population totale a reçu un questionnaire.

Comme le mentionne Poussart (2001), les erreurs attribuables à l'échantillonnage sont dues au fait que seule une partie de la population visée est jointe par le biais d'un échantillon aléatoire. Afin de limiter ce risque, nous nous sommes assurés d'inclure dans notre échantillon la totalité des PMCCA comportant plus d'un associé tout en nous dotant d'un échantillon important de praticiens exerçant seul. Afin d'éviter une surpondération de l'opinion des PMCCA comportant plus d'un associé en termes de représentativité au sein de l'échantillon par rapport à la population globale, nous avons pris soin, dans le cadre de l'analyse des résultats, de contrôler cette variable.

\subsection{Le questionnaire}

Le questionnaire a été conçu à la suite de la revue de la littérature réalisée en septembre 1998 et des entrevues réalisées auprès de certains commanditaires et des représentants de certains grands cabinets à cette même époque. Les rencontres avec des représentants de petits et de moyens cabinets ont permis de l'enrichir, de le préciser et de le synthétiser. Publiée en français, la version finale du questionnaire compte 65 questions.

Revue internationale P.M.E., vol. 15, n ${ }^{\text {s }} 3-4,2002$ 


\subsection{Le prétest}

Comme le relève Poussart (2001), un prétest a comme fonction principale d'évaluer la compréhension et la clarté du questionnaire auprès des répondants; il vise également à estimer le temps requis pour y répondre. Le prétest a été effectué en janvier 1999 auprès de 10 PMCCA établis dans toutes les régions de la province. Le prétest a permis de relever des imprécisions et d'apporter les modifications nécessaires. Un extrait du questionnaire est présenté en annexe.

\subsection{La collecte des données}

Les questionnaires ont été postés en juin 1999. Le délai entre le prétest et la mise à la poste de la version finale devait nous permettre d'éviter la période la plus occupée des PMCCA, soit la période d'impôt, qui débute en janvier et se termine normalement autour du $1^{\text {er }}$ mai. La date limite pour retourner le questionnaire a été fixée au 9 juillet 1999. Compte tenu des contraintes de temps et parce que les résultats de l'enquête correspondaient à la tendance déjà exprimée dans le prétest, nous n'avons pas effectué de rappel. En date du 31 juillet 1999, 124 questionnaires avaient été reçus; près de $8 \%$ de la population totale a donc répondu au questionnaire ${ }^{4}$.

Sans prétendre avoir contrôlé totalement le biais de non-répondant, nous avons néanmoins analysé l'ensemble des questionnaires reçus afin de nous assurer que le délai entre la date d'expédition et la date de réception des questionnaires ne pouvait être une source de biais. Entre le 8 août et le 9 septembre 1999, 23 questionnaires supplémentaires ont été reçus. L'analyse des résultats de ces 23 questionnaires indique qu'il n'existe pas de différences entre les réponses à ces questionnaires et les réponses aux 124 premiers questionnaires compilés. Au total, 147 questionnaires nous furent retournés pour un taux de réponse de 14,7\%.

\subsection{Le profil des répondants}

Les cabinets ayant participé à l'enquête comptent en moyenne 2,15 associés; le cabinet le plus important compte 12 associés. L'âge moyen des associés des PMCCA ayant participé à l'enquête est de 44 ans; l'âge des répondants varie entre 29 et 62 ans. En moyenne, nos répondants embauchent 4,93 professionnels et 1,94 employé (administratif); $47 \%$ d'entre eux sont des praticiens exerçant

4. À titre de comparaison, dans le cadre d'une étude sur l'adoption du commerce électronique par les PME québécoises, Poussart (2001) a sélectionné un échantillon de PME représentatives de la population correspondant à $0,5 \%$ de la population totale de PME québécoises, soit 1158 répondants sur un total de 222700 PME. Un nombre de réponse s'élevant à $8 \%$ de la population totale peut donc être considéré comme représentatif de la population globale.

Revue internationale P.M.E., vol. 15, nos 3-4, 2002 
seul, ce qui représentent $80,6 \%$ de tous les praticiens exerçant seul ayant répondu au questionnaire. Les répondants tirent en général $40 \%$ de leur chiffre d'affaires de la vérification et de l'examen d'états financiers, $23,5 \%$ de la comptabilité et $18 \%$ de la fiscalité. Les conseils en gestion ne comptent que pour $11 \%$ de leur chiffre d'affaires.

Au moment de l'enquête, les PMCCA possédaient, en moyenne, 2,39 ordinateurs portatifs et 6,73 ordinateurs de table et consacraient, en moyenne, $6,2 \%$ de leur chiffre d'affaires à la gestion de l'information. Ce pourcentage variait entre $0 \%$ et $30 \%$. Au total, $83 \%$ des répondants sont abonnés à Internet et $94 \%$ des répondants qui n'étaient pas abonnés comptaient le faire au cours de l'année.

Seulement $13 \%$ des répondants possédaient un site Web. Parmi les PMCCA qui n'ont pas de site Web, seulement 16,7\% ont l'intention d'en concevoir un. En moyenne, les répondants naviguent pendant 11 heures par mois, ce qui correspond à 5,11 heures par associé; certains affirment ne pas naviguer. En revanche, un cabinet comptant cinq associés a déclaré consacrer 120 heures à la navigation à des fins professionnelles. Les praticiens exerçant seul naviguent, en moyenne, 6,86 heures par mois. Dans les cabinets comptant plus de deux associés, on estime naviguer, en moyenne, pendant 14,86 heures par mois, soit une moyenne de 4,5 heures par associé.

Les moins de 40 ans $^{5}$ naviguent pendant 7,77 heures par mois en moyenne, ce qui signifie 4,6 heures par associé par mois alors que les 40 ans et plus naviguent pendant 12,18 heures par mois, ce qui représente environ 5,27 heures par associé. À notre grande surprise, ce sont donc ces derniers qui naviguent le plus.

\section{Les résultats de la recherche}

\subsection{L'attrait des modules proposés}

Parmi les 13 modules proposés, à la lecture du tableau 1, nous constatons que trois modules se distinguent assez nettement des autres. Ces trois modules sont Documentation officielle, Cybermanuel et Bases de connaissances et d'expertise (différence significative à $\mathrm{p}<0,001)^{6}$. Leur cote de popularité est respectivement

5. Deux raisons justifient le choix de l'âge de 40 ans comme ligne de démarcation au sein de la population étudiée. Premièrement, les plus de 40 ans n'utilisaient pas les technologies de l'information lorsqu'ils ont débuté en carrière, ce qui n'est habituellement pas le cas des moins de 40 ans. En effet, l'utilisation des ordinateurs personnels par les experts-comptables en cabinet a débuté autour de 1985. Deuxièmement, les tests statistiques effectués ont démontré des différences marquées entre ces deux populations.

6. Les tests de moyenne ont été effectués à l'aide du test «paired sample test» sur SPSS version 10.1.0. 
TABLEAU 1

Classement général des modules

\begin{tabular}{clccc}
\hline Rang & Cyberapplication & $\%$ & Moyenne & Écart type \\
\hline 1 & Documentation officielle & 85,7 & 6 & 1,27 \\
2 & Cybermanuel & 83,6 & 5,85 & 1,34 \\
3 & Bases de connaissances et d'expertise & 82,1 & 5,75 & 1,37 \\
4 & Service de recherche en ligne & 68,4 & 4,79 & 1,70 \\
5 & Formation virtuelle & 67,7 & 4,74 & 1,57 \\
6 & Boutique virtuelle & 64,9 & 4,54 & 1,65 \\
7 & Répertoires virtuels & 63,4 & 4,44 & 1,61 \\
8 & Applications transactionnelles & 62,9 & 4,40 & 1,71 \\
9 & Webdiffusion de nouvelles & 58,3 & 4,08 & 1,81 \\
10 & Marché virtuel & 55.0 & 3,86 & 1,70 \\
11 & Échanges entre CyberCA & 52,9 & 3,70 & 1,77 \\
12 & Certificats numériques & 50,4 & 3,53 & 1,82 \\
13 & Site Web du cabinet & 48,7 & 3,41 & 1,82 \\
\hline
\end{tabular}

Légende : Cote moyenne : 1 à 1,99 : pas intéressé ; 2 à 2,99 : plus ou moins intéressé ; 3 à 4,99 : moyennement intéressé ; 5 à 5,99 : intéressé; 6 à 7 : très intéressé.

Écart type: $\quad 0$ à 0,99 : consensus très élevé ; 1 à 1,49 : consensus élevé ; 1,49 à 1,99 : consensus faible; plus de 2 : consensus très faible.

Sources : Berry, 1978; Berry, Harwood et Katz, 1987.

de $85,7 \%, 83,6 \%$ et $82,1 \%$. En utilisant les critères de classification de Berry (1978 ; Berry, Harwood et Katz, 1987), l'écart type pour ces trois modules indique un degré de consensus très élevé. Ces modules présentent la particularité d'offrir un contenu en information à valeur ajoutée élevée. Par conséquent, il semble clair que, pour les PMCCA, l'aspect information est nettement plus important que les aspects de mise en relation ou encore transactionnels.

Cinq modules ne semblent pas intéresser les PMCCA (différence significative à $\mathrm{p}<0,05)$. Premièrement, le module Site Web, qui s'est classé au dernier rang. Seulement $10,4 \%$ des répondants utiliseront certainement (7/7) ce module alors que $15,3 \%$ ne l'utiliseront pas du tout (1/7). Ce résultat est cohérent avec ce que nous avons déjà observé de la situation actuelle des PMCCA. On se rappellera que $87 \%$ des PMCCA n'ont pas de site Web. Seulement 16,67\% d'entre eux ont l'intention de se doter d'un site Web au cours de la prochaine année. Pourquoi les PMCCA sont-ils réticents à s'établir sur le Web ? Considèrent-ils que c'est une perte de temps, que leurs clients ne sont pas intéressés, ou encore qu'ils n'ont rien à apporter de neuf à la communauté ? Deuxièmement, le module Certificats numériques, qui sera utilisé certainement (7/7) par 9,8\% des PMCCA alors que $14,6 \%$ des PMCCA ne l'utiliseront pas (1/7). Troisièmement, concernant le module qui facilite les Échanges entre CyberCA, $8 \%$ des PMCCA cyberbavarderont certainement (7/7) alors que $12 \%$ ne le feront pas du tout (1/7). Quatrièmement, $9 \%$ des PMCCA utiliseront le module Marché virtuel (7/7) alors que $5 \%$ n'y sont

Revue internationale P.M.E., vol. 15, $\mathrm{n}^{\text {os }} 3-4,2002$ 
pas intéressés (1/7). Cinquièmement, le module Webdiffusion de nouvelles n'intéresse réellement que $12 \%$ des PMCCA qui l'utiliseront (7/7) alors que $9 \%$ ne l'utiliseront pas du tout (1/7). Pour ces cinq modules, l'écart type indique un degré de consensus élevé. Ces modules portent essentiellement sur les dimensions relationnelles et transactionnelles.

\subsection{Incidence du nombre d'associés}

Nous nous sommes demandé si l'intérêt pour le cyberespace est influencé par le nombre d'associés œuvrant au sein du cabinet. Pour répondre à la question, nous avons segmenté la population totale en deux sous-populations : la première regroupe les cabinets comptant un seul associé; la seconde, les cabinets comptant deux associés et plus. On croyait, par exemple, que les praticiens exerçant seul seraient plus attirés par les Échanges entre CyberCA parce qu'ils y trouveraient des interlocuteurs auprès desquels ils pourraient peaufiner leur jugement. Si tel était le cas, toutes les applications qui leur permettraient d'élargir leur réseau devraient les attirer.

Le tableau 2 compare les cotes attribuées par chacune des sous-populations. Globalement, les deux sous-populations réagissent de façon similaire. Le test de Student qui permet de comparer les moyennes entre les deux catégories s'est révélé non significatif.

\subsection{Incidence de l'âge des associés}

Nous nous sommes demandé également si l'âge moyen des associés œuvrant au sein du cabinet avait une incidence sur l'intérêt pour le cyberespace. Nous croyions que plus le praticien serait âgé, plus faible serait son intérêt pour le cyberespace.

Pour répondre à la question, nous avons divisé la population totale en deux sous-populations : la première regroupe les cabinets dont la moyenne d'âge des associés est inférieure à 40 ans; la seconde, les cabinets dont les associés ont 40 ans et plus en moyenne. Le tableau 3 compare les résultats des deux groupes. A priori, on peut conclure que l'âge des associés a une incidence sur leur intérêt pour le cyberespace. À part le module Documentation officielle (86,1\% vs $84,7 \%$; $\mathrm{p}<0,05)$ qui attire davantage les 40 ans et plus que les autres et le module Site Web $(54,4 \%$ vs $46,7 \% ; \mathrm{p}<0,01)$ qui attire davantage les moins de 40 ans que les autres, tous les autres modules sont légèrement mieux accueillis dans la population des moins de 40 ans.

Dans cette sous-population composée des associés de moins de 40 ans, les trois premières places ont été attribuées respectivement aux modules Bases de connaissances et d' expertise (87\%), ex-aequo avec le module Cybermanuel (87\%); Documentation officielle $(84,7 \%)$ et au module Formation virtuelle $(75,3 \%)$.

Revue internationale P.M.E., vol. 15, ns 3-4, 2002 
TABLEAU 2

Préférences en fonction du nombre d'associés (en pourcentage)

\begin{tabular}{lcc}
\hline Cyberapplication & 1 associé & 2 et plus \\
\hline Documentation officielle & 85,9 & 85,7 \\
Cybermanuel & 83,6 & 83,6 \\
Bases de connaissances et d'expertise & 82,1 & 82,1 \\
Service de recherche en ligne & 70,1 & 66,6 \\
Formation virtuelle & 68,6 & 66,7 \\
Boutique virtuelle & 68,4 & 61,3 \\
Répertoires virtuels & 65,6 & 61,3 \\
Applications transactionnelles & 64,4 & 61,3 \\
Webdiffusion de nouvelles & 54,7 & 61,7 \\
Échanges entre CyberCA & 53,6 & 52,0 \\
Marché virtuel & 52,7 & 57,6 \\
Certificats numériques & 49,0 & 50,9 \\
Site Web & 47,0 & 50,6 \\
\hline
\end{tabular}

TABLEAU 3

Préférences en fonction de l'âge des associés (en pourcentage)

\begin{tabular}{lcc}
\hline Cyberapplication & moins de $\mathbf{4 0}$ ans & $\mathbf{4 0}$ ans et plus \\
\hline Bases de connaissances et d'expertise & 87,0 & 80,4 \\
Cybermanuel & 87,0 & 82,3 \\
Documentation officielle* & 84,7 & 86,1 \\
Formation virtuelle & 75,3 & 64,9 \\
Service de recherche en ligne & 74,9 & 66,0 \\
Boutique virtuelle & 73,6 & 61,7 \\
Répertoires virtuels & 70,4 & 60,9 \\
Applications transactionnelles & 66,6 & 61,4 \\
Webdiffusion de nouvelles & 64,9 & 55,9 \\
Marché virtuel & 64,9 & 52,0 \\
Échanges entre CyberCA & 63,6 & 48,9 \\
Certificats numériques & 55,3 & 48,5 \\
Site Web** & 54,4 & 46,7 \\
\hline
\end{tabular}

$* \mathrm{p}<0,05$.

$* * \mathrm{p}<0,01$

Dans la sous-population des associés de plus de 40 ans, les répondants ont placé, dans l'ordre, les modules Documentation officielle, Cybermanuel et Bases de connaissances et d'expertise aux trois premiers rangs. Ce classement diffère de celui des moins de 40 ans qui font preuve de plus d'audace dans leurs choix en accordant la première place aux Bases de connaissances et d'expertise et au Cybermanuel, deux nouveaux produits pour lesquels ils ont peu de repères. 


\subsection{La valeur des modules proposés}

Dans la première section du questionnaire, les répondants ont exprimé leur intérêt pour les 13 modules qui seront à la base de la communauté virtuelle. Dans la section II du questionnaire d'enquête, ils ont été invités à estimer les sommes annuelles qu'ils seraient prêts à débourser pour avoir accès à @BACUS ${ }^{\mathrm{TM}}$.

Le prétest avait permis de constater qu'il était difficile de placer un chiffre sur la valeur de l'abonnement annuel à une communauté virtuelle de même que sur l'abonnement mensuel aux divers modules; plusieurs répondants ne répondaient tout simplement pas. Des modifications ont été apportées au questionnaire d'enquête définitif afin de réduire le nombre d'estimations requises et ainsi de permettre aux répondants de se concentrer sur les évaluations les plus significatives pour eux.

En moyenne, les PMCCA seraient prêts à verser $714 \$$ annuellement pour accéder à @BACUS ${ }^{\mathrm{TM}}$, ou $332 \$$ par associé7. Le tableau 4 synthétise toutes les appréciations des PMCCA. Fait intéressant, il existe une relation positive et significative entre le nombre d'ordinateurs au sein du PMCCA et le débours que les cabinets sont prêts à assumer (corr. : 0,386, $0<0,001$ ). Par conséquent, on peut penser que lorsque l'utilisation des ordinateurs personnels est répandue au sein des cabinets, les associés sont prêts à investir des montants plus substantiels pour adhérer à une communauté virtuelle.

Quelle valeur les praticiens exerçant seul attribuent-ils à @BACUSTM ? Leurs ressources financières souvent plus limitées que celles des cabinets comptant plus d'un associé ont-elles une incidence sur la valeur accordée? Leur isolement en a-t-il un? L'analyse des données montre que les praticiens exerçant seul qui ont répondu à la question seraient prêts à verser 513 \$ par année. De leur côté, les cabinets comptant plus d'un associé seraient prêts à verser 910 \$ en moyenne pour le cabinet, ou $276 \$$ par associé (différence significative à $\mathrm{p}<0,001$ ).

Dans la section précédente, il est clairement ressorti que les moins de 40 ans sont plus intéressés que les 40 ans et plus par le concept de communauté virtuelle. L'âge a-t-il aussi un effet sur la valeur que les PMCCA donnent à @BACUS ${ }^{\mathrm{TM}}$ ? L'analyse des données révèle qu'en moyenne, par associé, les PMCCA dont les associés ont moins de 40 ans sont prêts à verser $456 \$$ par année par associé pour utiliser@BACUS ${ }^{\mathrm{TM}}$. Ceux dont l'âge moyen est de 40 ans ou plus seraient prêts à verser $300 \$$ par associé pour y accéder (différence significative à $\mathrm{p}<0,05$ ).

7. À cette question, 95 PMCCA ont répondu, soit 76,6\% des répondants. Dans le calcul des valeurs moyennes, la valeur 0 n'a pas été attribuée par défaut aux questionnaires des non-répondants. La moyenne a donc été compilée à partir des 95 réponses reçues et a exclu les non-répondants.

Revue internationale P.M.E., vol. 15, n ${ }^{\text {s }} 3-4,2002$ 
Les répondants ont également été invités à désigner les cinq modules qui les intéressent davantage ; de plus, ils devaient préciser le montant qu'ils étaient prêts à débourser pour les utiliser. Ces réponses exigent des PMCCA qu'ils poussent plus loin l'exercice entrepris dans la section I du questionnaire. Il est facile d'affirmer qu'un module est intéressant; le choix devient plus sérieux lorsqu'on doit préciser le déboursé que l'on accepte de faire pour l'utiliser.

TABLEAU 4

Synthèse comparative de l'appréciation des PMCCA

\begin{tabular}{lccccc}
\hline $\begin{array}{l}\text { Abonnement } \\
\text { annuel }\end{array}$ & $\begin{array}{c}\text { Montant } \\
\text { minimal } \\
\$\end{array}$ & $\begin{array}{c}\text { Montant } \\
\text { maximal } \\
\$\end{array}$ & $\begin{array}{c}\text { Moyenne } \\
\text { globale } \\
\$\end{array}$ & $\begin{array}{c}\text { Moyenne Répondants } \\
\text { par associé } \\
\$ \$\end{array}$ & \\
\hline $\begin{array}{l}\text { Population totale } \\
\text { Praticiens exerçant seul* }\end{array}$ & 25 & 3500 & 714 & 332 & 95 \\
$\begin{array}{l}\text { Cabinet de 2 associés } \\
\text { et plus* }\end{array}$ & 25 & 1500 & 513 & 513 & 47 \\
$\begin{array}{l}\text { Moins de 40 ans** } \\
\text { 40 ans et plus** }\end{array}$ & 200 & 3500 & 910 & 276 & 48 \\
\hline
\end{tabular}

* Différence de moyenne significative à $\mathrm{p}<0,001$

** Différence de moyenne significative à $\mathrm{p}<0,05$.

Encore une fois les PMCCA ont affiché leur préférence pour tous les modules à contenu informationnel, soit les modules Documentation officielle, Bases de connaissances et d'expertise, Cybermanuel, Service de recherche en ligne et Formation virtuelle (tableau 5).

Le module Service de recherche en ligne offre un service de rédaction d'opinions professionnelles sur des questions de comptabilité, de vérification et de fiscalité. Ce service est rémunéré à l'heure. Parmi les PMCCA qui ont indiqué le taux qu'ils seraient prêts à accepter, le taux minimal proposé est de 60 1'heure et le taux maximal est de $100 \$$ l'heure; le taux moyen serait de 77,50 \$ l'heure.

Les PMCCA ont été invités à exprimer le montant qu'ils étaient prêts à payer pour un cours virtuel. Le débours moyen par cours s'élève à 114,28 \$. Le déboursé le plus faible est de 50 \$ par cours et le plus élevé, de 200 \$ par cours. Deux répondants n'ont pas procédé à l'estimation. Cependant, ils ont mentionné qu'ils étaient prêts à payer les mêmes tarifs que ceux qui sont appliqués pour un cours régulier offert par l'Ordre des comptables agréés du Québec.

Notre analyse a démontré que le nombre d'associés œuvrant au sein du cabinet n'a pas eu d'incidence sur le choix des modules; le palmarès établi par les deux groupes est le même. Cependant, il semble que, toutes proportions gardées, les praticiens exerçant seul seraient prêts à débourser davantage pour utiliser les modules. Par exemple, les praticiens exerçant seul seraient prêts à verser, en moyenne, 138 \$

Revue internationale P.M.E., vol. 15, nos 3-4, 2002 
pour utiliser la Documentation officielle alors que les cabinets de plus de deux associés, seraient prêts à verser 91 \$ en moyenne par associé (différence significative à $\mathrm{p}<0,001)$.

Dans le même ordre d'idées, il semble que l'âge moyen a une certaine incidence sur les débours acceptés. Les moins de 40 ans seraient prêts à débourser plus que les 40 ans et plus pour utiliser les modules proposés. Par exemple, alors qu'un associé ayant moins de 40 ans serait prêt à verser, en moyenne, 280 \$ pour utiliser la Documentation officielle, celui qui a 40 ans ou plus verserait $178 \$$ $(\mathrm{p}<0,06)$.

TABLEau 5

Les cinq modules ayant le plus de valeur au sein de la population totale des PMCCA

\begin{tabular}{lccc}
\hline Module & $\begin{array}{c}\text { Débours } \\
\text { total }\end{array}$ & $\begin{array}{c}\text { Débours } \\
\text { minimal }\end{array}$ & $\begin{array}{c}\text { Débours } \\
\text { maximal }\end{array}$ \\
\hline Documentation officielle* $^{*}$ ' & $207 \$$ & $10 \$$ & $1000 \$$ \\
Bases de connaissance et d'expertise* & $173 \$$ & $10 \$$ & $1000 \$$ \\
Cybermanuel* & $113 \$$ & $10 \$$ & $800 \$$ \\
Service de recherche en ligne & $79 \$ /$ heure & $5 \$$ & $250 \$$ \\
Formation virtuelle & $142 \$ /$ cours & $25 \$$ & $500 \$$ \\
\hline
\end{tabular}

* Différence de moyenne significative à $\mathrm{p}<0,001$.

\subsection{La position des PMCCA à l'égard du développement et de l'exploitation de la communauté virtuelle}

Dans la troisième section du questionnaire, les répondants devaient préciser quelle entité, à leur avis, serait la mieux placée pour concevoir et pour exploiter une communauté virtuelle destinée aux PMCCA. Selon Granovetter (1985), il est important que les membres de la communauté aient confiance dans le maître d'œuvre. Parallèlement, le maître d'œuvre doit également en tirer des avantages. Comme le mentionnent Rotharermel et Sugiyama (2001), une communauté virtuelle gérée efficacement engendre des bénéfices économiques autant pour ses membres que pour le maître d'œuvre qui exploite la communauté virtuelle. Dans l'ensemble, comme le montre le tableau 6, 42,9\% des répondants sont d'avis que l'Ordre des comptables agréés du Québec (OCAQ), qui est l'ordre représentant les intérêts de la profession de comptable agréé, devrait être le maître d'œuvre de la communauté virtuelle ; $21 \%$ pensent que cette tâche devrait être confiée à une université ; $9 \%$ croient qu'un des leurs, soit un PMCCA, devrait être le maître d'œuvre de la communauté virtuelle; $6 \%$ croient qu'une firme de conseillers en informatique devrait être le maître d'œuvre. Vingt et un pourcent des répondants croient 
qu'@BACUS ${ }^{\mathrm{TM}}$ devrait être conçu et exploité par une autre entité. Parmi ceux qui croient qu'une autre entité serait mieux placée pour assumer la maîtrise d'œuvre, $88 \%$ (ou 17,6\% de l'ensemble des répondants) proposent une forme quelconque de partenariat. La plus populaire est celle qui réunit l'OCAQ et une université $(28 \%)$.

Notre analyse a montré que le nombre d'associés œuvrant au sein du cabinet n'a pas eu d'incidence sur le choix du maître d'œuvre. Toutefois, on note des différences considérables au niveau de l'âge des répondants. Seulement 34,4\% des moins de 40 ans estiment que l'OCAQ devrait assumer la maîtrise d'œuvre d'@BACUS ${ }^{\mathrm{TM}}$. En revanche, ils sont $28,1 \%$ à croire que c'est une université qui devrait concevoir et exploiter la communauté virtuelle; $18,8 \%$ d'entre eux suggèrent une autre entité. Pour leur part, $46 \%$ des plus de 40 ans, soit la plus forte proportion, confieraient la maîtrise d'œuvre à l'OCAQ; 18,4\% choisiraient une université et $21,3 \%$, une autre entité.

\subsection{Le financement de la communauté virtuelle}

Dans cette sous-section du questionnaire d'enquête, les répondants étaient invités à choisir le mode de financement d'@BACUS ${ }^{\mathrm{TM}}$ leur convenant le mieux. Les répondants n'avaient pas à limiter leur choix à une solution; $10 \%$ des répondants ont choisi plus d'un mode.

Les données ont été analysées pour la population totale, en fonction du nombre d'associés et de l'âge moyen des associés (analyse horizontale); elles ont aussi été analysées en fonction des choix proposés (analyse verticale).

Comme le montre le tableau 7, le mode de financement préféré dans une proportion de 49,2\% de la population totale est l'abonnement annuel donnant accès à certaines sections jumelées à un accès à la carte pour les modules plus coûteux (abonnement annuel jumelé à un accès à la carte). C'est l'accès à la carte qui est le moins populaire : il a fait partie de $12,9 \%$ des choix. Le nombre d'associés a une incidence sur les préférences. Ainsi l'abonnement annuel accompagné d'un accès à la carte pour les modules plus coûteux est le choix qui convient le mieux aux cabinets comptant deux associés et plus alors que les autres modes de paiement intéressent davantage les praticiens exerçant seul.

L'âge a encore beaucoup d'influence sur les choix. L'abonnement annuel jumelé à un accès à la carte attire les plus de 40 ans dans une proportion de $53,2 \%$, par rapport à $39,5 \%$ chez les moins de 40 ans. Dans la population totale, cette proportion est de 49,2\%. L'accès à la carte n'est pas un choix qui convient aux plus de 40 ans; seulement 10,6\% d'entre eux l'ont choisi, par rapport à 18,4\% des moins de 40 ans et à 12,9\% de la population totale.

Revue internationale P.M.E., vol. 15, nos 3-4, 2002

(C) 2003 - Presses de l'Université du Québec

Édifice Le Delta I, 2875, boul. Laurier, bureau 450, Sainte-Foy, Québec G1V 2M2 • Tél. : (418) 657-4399 - www.puq.uquebec.ca

Tiré de : Revue internationale P.M.E., vol. 15, nos $3-4$, sous la direction de Pierre-André Julien. 
TABLEAU 6

Qui devrait concevoir et exploiter la communauté virtuelle

\begin{tabular}{lcllcc}
\hline & PMCCA & OCAQ & Université & TI & Autres \\
\hline Population totale & $9,2 \%$ & $42,9 \%$ & $21,0 \%$ & $5,9 \%$ & $21,0 \%$ \\
Praticiens exerçant seul & $8,2 \%$ & $42,6 \%$ & $18,0 \%$ & $6,6 \%$ & $24,6 \%$ \\
Cabinet de 2 associés & & & & & \\
$\quad$ et plus & $10,4 \%$ & $43,1 \%$ & $24,1 \%$ & $5,2 \%$ & $17,2 \%$ \\
Moins de 40 ans & $9,6 \%(1)$ & $34,4 \%{ }^{(2)}$ & $28,1 \%(3)$ & $3,1 \%$ & $18,8 \%$ \\
40 ans et plus & $6,9 \%(1)$ & $46 \%{ }^{(2)}$ & $18,4 \%{ }^{(3)}$ & $6,9 \%$ & $21,8 \%$ \\
\hline
\end{tabular}

Différences de moyennes significatives à (1) $\mathrm{p}<0,01$ (2) $\mathrm{p}<0,05$ (3) $\mathrm{p}<0,05$.

TABLEAU 7

Comment financer la communauté virtuelle

\begin{tabular}{|c|c|c|c|c|}
\hline & $\begin{array}{l}\text { Accès } \\
\text { à la carte }\end{array}$ & $\begin{aligned} & \text { Abonnement } \\
+ & \text { accès à la carte }\end{aligned}$ & $\begin{array}{l}\text { Abonnement } \\
\text { global }\end{array}$ & $\begin{array}{l}\text { Cotisation } \\
\text { spéciale* }^{*}\end{array}$ \\
\hline Population totale & $12,9 \%$ & $49,2 \%$ & $19,3 \%$ & $20,5 \%$ \\
\hline Praticiens exerçant seul & $15,3 \%$ & $40,3 \%$ & $20,8 \%$ & $23,6 \%$ \\
\hline $\begin{array}{l}\text { Cabinet de } 2 \text { associés } \\
\text { et plus (1) }\end{array}$ & $10,0 \%(1)$ & $60,0 \%$ & $13,3 \%$ (2) & $16,7 \%$ \\
\hline Moins de 40 ans & $18,4 \%$ & $39,5 \%$ & $15,8 \%$ & $26,3 \%$ \\
\hline 40 ans et plus & $10,6 \%$ & $53,2 \%$ & $18,1 \%$ & $18,1 \%$ \\
\hline
\end{tabular}

Différences de moyennes significatives à (1) $\mathrm{p}<0,01(2) \mathrm{p}<0,001$.

* Cotisation spéciale obligatoire établie en fonction du nombre d'associés du cabinet.

\section{Conclusion}

Les résultats de notre recherche ont démontré le grand intérêt des PMCCA à l'égard d'une communauté virtuelle dans la mesure où les coûts liés à son utilisation offrent un bon rapport qualité-prix. Les modules les plus appréciés par les PMCCA sont sans conteste les modules à fort contenu en information tels que la Documentation officielle, le Cybermanuel et les Bases de connaissances et d'expertise.

Comme les PMCCA sont prêts à débourser annuellement 714 \$, dans l'hypothèse où $50 \%$ des quelque 1600 PMCCA actuels du Québec s'abonneraient à la communauté virtuelle, son promoteur disposerait d'un budget de près de $600000 \$$ annuellement. Toutefois, le défi est de taille, car les PMCCA sont très sensibles au rapport qualité-prix et aux économies de temps qu'ils peuvent réaliser. Dans une certaine mesure, tous les services offerts par la communauté virtuelle devraient éventuellement pouvoir être facturés aux clients. En conclusion, les PMCCA sont intéressés aux modules qui leur permettent d'ajouter de la valeur aux services qu'ils rendent à leurs clients. 
Cependant, il nous a semblé que les PMCCA ne prenaient pas pleinement conscience du pouvoir d'achat et de la valeur ajoutée que peut leur apporter un regroupement au sein d'une communauté virtuelle. Pourtant, le fossé qui ne cesse de se creuser entre les ressources et les services offerts par les grands cabinets d'experts-comptables et les PMCCA ainsi que la menace concrète que font planer sur eux certains nouveaux fournisseurs de services, autrefois étrangers au domaine des services comptables, devraient inquiéter les PMCCA. Ce n'est qu'en prenant réellement conscience de ces menaces qu'ils seront prêts à investir des montants substantiels dans une communauté virtuelle qui constitue, pour eux, une façon de demeurer compétitif dans un marché de plus en plus concurrentiel.

\section{Les limites de la recherche}

Dans le cadre du processus de validation du concept de sites Web, de portail ou de communauté virtuelle, il est toujours souhaitable de demander aux répondants d'apprécier la valeur du produit qui leur est présenté à partir d'un prototype. En raison de considérations techniques et financières hors de notre contrôle, l'évaluation de la communauté virtuelle a dû être effectuée à partir d'une description narrative du contenu des modules. Si les répondants avaient eu la possibilité de consulter un prototype ne serait-ce que partiel, il est probable que les résultats de cette recherche auraient été quelque peu différents des résultats obtenus par le biais de l'enquête par questionnaire.

Dans un processus rigoureux d'identification des besoins, l'objectif est non seulement d'identifier les besoins actuels mais également les besoins émergents ou latents. Dans le cadre de notre recherche, le processus d'entrevue élargi auprès des grands cabinets et des fournisseurs de services que nous avons appliqué avait pour objectif de faciliter l'identification des besoins que les PMCA n'étaient pas en mesure d'exprimer clairement. Toutefois, malgré tout le soin apporté à la définition des besoins, il est possible que notre cadre conceptuel soit incomplet et qu'il ait négligé des fonctionnalités qui auraient été importantes et qui auraient possiblement influencé l'intérêt porté aux autres modules. De plus, le potentiel de la communauté virtuelle proposée a été évalué à la lumière des technologies disponibles au moment de l'enquête. L'émergence de toute nouvelle technologie peut modifier considérablement les besoins des utilisateurs potentiels dans le temps.

Le concept de communauté virtuelle proposé dans cet article est un modèle logique. Ces modèles, à l'instar des modèles physiques, ne tiennent pas compte des contraintes politiques, technologiques et économiques pouvant influencer le développement d'une communauté virtuelle viable et prospère. Il est évident que certaines contraintes risquent d'influencer le développement de certains modules proposés ou de leurs fonctionnalités.

Revue internationale P.M.E., vol. 15, nos 3-4, 2002 


\section{Avenue de recherche}

L'une des questions qui mériterait d'être validée dans le cadre de recherches ultérieures est celle-ci : dans quelle mesure le concept de communauté virtuelle proposé dans cet article peut être extrapolable à d'autres professions? Dans la mesure où ces professions offrent des services professionnels qui requièrent d'importantes connaissances tels que les services juridiques (notaires et avocats) et les servicesconseils (architectes, ingénieurs, conseillers en systèmes d'information), nous croyons que plusieurs des modules proposés ici pourraient présenter un intérêt. Toutefois, chaque profession a ses besoins particuliers et la nature ainsi que l'importance relative des modules proposés peut varier d'une profession à l'autre. Par conséquent, nous pensons que seules une étude approfondie et une analyse comparative des besoins des diverses professions libérales pourraient apporter une réponse définitive à cette question.

En conclusion, peu importe les questions d'ordre théorique, méthodologique et pratique qui se posent dans le cadre d'une telle recherche-action, ce sont les futurs membres de la communauté virtuelle qui auront le dernier mot. Nous espérons cependant que la démarche scientifique adoptée pour l'identification des besoins a contribué à réduire considérablement les risques associés à un tel projet.

\section{ANNEXE I \\ Extrait du questionnaire @BACUS ${ }^{\mathrm{TM}}$, la communauté virtuelle des PMCCA}

Imaginez un site Web - dans Internet - qui soit expressément conçu pour un cabinet comme le vôtre. Ce site vous donnerait accès à une foule de renseignements, comme des lois et des formulaires, ainsi qu'à des outils informatisés - logiciels ou bases de données - qui faciliteraient votre travail, vous rendraient plus compétitifs et vous permettraient d'ajouter de la valeur aux services que vous rendez déjà à vos clients. Ce site Web, c'est @BACUS ${ }^{\mathrm{TM}}$, la communauté virtuelle des PMCCA.

\section{Que contient @BACUSTM}

$@$ BACUS ${ }^{\mathrm{TM}}$ compte treize modules (référez-vous au plan présenté ci-contre). Pour chacun d'entre eux, nous voulons mesurer votre intérêt. Donc, encerclez la cote qui le décrit le mieux $(1=$ aucun intérêt ; 7 = prêt à l'utiliser). Précisez aussi le montant que vous voulez investir sur une base annuelle pour y accéder.

\section{Documentation officielle}

Vous utilisez la réglementation fiscale, le Manuel de l'ICCA et, à l'occasion, des lois des compagnies. Vous avez besoin des mises à jour du Guide du vérificateur des municipalités? Vous voulez consulter des Guides d'application pratique des nouvelles normes comptables, rédigés par des spécialistes de recherche, qui traitent des vrais problèmes? Vous utilisez quotidiennement des programmes de vérification ou d'examen, des modèles de demande de confirmation et des formulaires fiscaux? Où trouver un exemple de lettre de mission de vérification toujours à jour? Finies les recherches infructueuses, les mises à jour fastidieuses et la gestion d'un entrepôt de formulaires. Remplacez votre bibliothèque par le module Documentation officielle et retrouvez, au même endroit, des textes et des outils toujours à jour. 
Pas du tout

Utiliserez-vous

le module Documentation officielle?

Combien êtes-vous prêt à investir annuellement?
Certainement

$$
\begin{array}{lllllll}
1 & 2 & 3 & 4 & 5 & 6 & 7
\end{array}
$$

\section{Bases de connaissances et d'expertise}

Savez-vous où trouver les états financiers d'une société prête-nom? Des exemples de notes décrivant des opérations de troc? Vous voulez consulter facilement une banque d'états financiers de sociétés ouvertes? Vous voulez accéder aux archives publiques, comparer les états financiers de votre client avec des ratios vraiment pertinents ou avoir des exemples de recommandations portant sur des lacunes du contrôle interne afin de rédiger plus rapidement votre lettre sur le contrôle interne ? Un client souhaite connaître les meilleures pratiques connues de gestion des comptes clients et vous ne savez pas où chercher? Où trouver une banque consolidée des subventions offertes aux PME et aux OSBL pour aider votre client à trouver les fonds dont il a besoin ? Le module Bases de connaissances et d'expertise d'@BACUS ${ }^{\mathrm{TM}}$ contient la banque de données dont vous avez besoin.

Pas du tout

Utiliserez-vous

les Bases de connaissances et d' expertise?

Combien êtes-vous prêt à investir annuellement ?
Certainement

$\begin{array}{lllllll}1 & 2 & 3 & 4 & 5 & 6 & 7\end{array}$

\section{Cybermanuel de l'ICCA}

Vous rêvez d'un Manuel de l'ICCA enrichi, comportant des commentaires pratiques, des renvois aux articles pertinents du Code civil ou de la Loi de l'impôt? Vous aimeriez y retrouver des exemples de notes aux états financiers, des définitions, des modèles de lettres de demande de confirmation prêts à être utilisés ou des liens aux autres chapitres pertinents? Le Cybermanuel contient des hyperliens à tous ces renseignements.

\section{Pas du tout}

Certainement

Utiliserez-vous le Cybermanuel de l' ICCA?

Combien êtes-vous prêt à investir annuellement?

\section{$4 \quad$ Service de recherche en ligne}

Vous avez besoin d'un avis écrit sur une question comptable ou fiscale? Soumettez votre problème par courrier électronique à l'aide d'un formulaire de demande d'opinion de recherche prédéfini. Un spécialiste de recherche, qui connaît bien les PME, rédigera une opinion de recherche qu'il vous retournera aussi par courrier électronique, après vous avoir fait part de son estimation du temps à y consacrer.

\section{Pas du tout}

Certainement

Utiliserez-vous le Service de recherche en ligne?

12

3

Quel taux horaire êtes-vous prêt à accorder pour ce service? 


\section{$5 \quad$ Applications transactionnelles}

@BACUS ${ }^{\mathrm{TM}}$ vous propose des applications qui vous permettront de tirer profit des possibilités infinies d'Internet. Ainsi, ne postez plus de confirmations bancaires. Courriellez-les et économisez un temps précieux à toutes les étapes du processus. Vous ne savez pas où conserver vos sauvegardes? Transférez vos fichiers par Internet à @BACUSTM qui les entreposera sur ses équipements. Courriellez des questions aux représentants du ministère du Revenu au sujet de la situation fiscale d'un client ou aidez votre client à obtenir, en ligne, une majoration de sa marge de crédit.

Pas du tout

Utiliserez-vous

les Applications transactionnelles?

\section{2}

Certainement

\section{$\begin{array}{ll}6 & 7\end{array}$}

Combien êtes-vous prêt à investir annuellement?

\section{Certificats numériques (signatures électroniques)}

La confidentialité de vos échanges électroniques vous préoccupe? Vous voulez signer électroniquement les messages destinés à votre client ? Vous voulez courrieller ses états financiers à votre client et celui-ci se questionne sur la sécurité du transfert ? Codez les états financiers au moyen d'une clé (encryption). Votre client les décodera de la même manière. @BACUS ${ }^{\mathrm{TM}}$ vous fournira une clé. Il y en aura aussi une pour votre client. Celui-ci pourra même suivre un cours dans Internet pour apprendre à s'en servir.

\section{Pas du tout}

Certainement

Utiliserez-vous un Certificat numérique?

$\begin{array}{lllllll}1 & 2 & 3 & 4 & 5 & 6 & 7 \\ 1 & 2 & 3 & 4 & 5 & 6 & 7\end{array}$

Utiliserez-vous l'encryption?

Combien êtes-vous prêt à payer pour avoir un certificat numérique ?

Combien êtes-vous prêt à payer pour avoir un certificat numérique ?

\section{$7 \quad$ Boutique virtuelle}

Qu'il s'agisse de tenue de livres, de vérification, de déclaration d'impôts ou de tableurs, la boutique virtuelle d'@BACUS ${ }^{\mathrm{TM}}$ a le logiciel dont vous avez besoin. Son moteur de recherche vous permet de trouver rapidement ce que vous cherchez. Vous pouvez télécharger le logiciel, le tester et profiter d'un rabais par rapport au prix régulier. Vous hésitez entre deux produits? Lisez des évaluations indépendantes avant de choisir. Après l'achat, vous avez des commentaires à formuler? Faites connaître votre opinion aux autres membres de la communauté virtuelle. La boutique offre aussi des ordinateurs.

\section{Pas du tout}

Utiliserez-vous la Boutique virtuelle?

À quel montant s'élèveront vos achats annuels ?

\section{$8 \quad$ Site Web de votre cabinet}

Accroissez votre visibilité sur le Net. Montez votre site Web en quinze minutes et @BACUSTM l'hébergera. Tous les visiteurs de la communauté pourront y faire un tour. Si vous le souhaitez, un spécialiste peut concevoir un site Web plus étoffé qui satisfait à vos exigences spécifiques. 


\section{Pas du tout}

Utiliserez-vous le module Site Web?

Combien êtes-vous prêt à investir annuellement pour l'hébergement de votre site Web?

Combien êtes-vous prêt à investir pour la conception personnalisée de votre site Web?

\section{$9 \quad$ Webdiffusion (push) de nouvelles}

Aimeriez-vous recevoir des nouvelles des secteurs d'activité ou des régions qui vous intéressent, des nouvelles financières, les cotes boursières ou les fluctuations du huard? Faites vos choix et voyez défiler les nouvelles sur l'écran de votre ordinateur.

\section{Pas du tout}

Certainement

Utiliserez-vous la Webdiffusion de nouvelles?

1

23

4

5

Combien êtes-vous prêt à investir annuellement ?

10 Échanges entre CyberCA

Vous voulez participer à des forums avec des pairs? Peut-être voulez-vous échanger plus directement avec un $\mathrm{CA}$ qui a résolu un problème comme celui que vous pose un client? $@ \mathrm{BACUS}^{\mathrm{TM}}$ réunit des $\mathrm{CA}$ comme vous, prêts à cyberpartager.

\section{Pas du tout}

Utiliserez-vous

le module Échanges entre CyberCA?

Combien êtes-vous prêt à investir annuellement?
Certainement

$$
\begin{array}{lllllll}
1 & 2 & 3 & 4 & 5 & 6 & 7
\end{array}
$$

\section{Répertoires virtuels}

Rien n'est plus frustrant que de chercher longuement quelque chose dans Internet. Facilitez vos recherches avec les répertoires virtuels. Un client se questionne sur la fiscalité américaine ou fait face à un problème comptable très complexe ? Consultez le Réseau de compétences. $@$ BACUS $^{\mathrm{TM}}$ vous offrira aussi le Bottin des représentants du ministère du Revenu et celui des cybermembres de l'OCAQ. Retrouvez la Liste des entreprises québécoises branchées ou naviguez dans Internet, à l'aide du Répertoire convivial des bonnes adresses Internet.

\section{Pas du tout}

Certainement

Utiliserez-vous les Répertoires virtuels?

$$
12
$$

\section{$\begin{array}{lll}5 & 6 & 7\end{array}$}

Combien êtes-vous prêt à investir annuellement ?

\section{Marché virtuel}

Votre client recherche des partenaires commerciaux aux États-Unis ou encore, il veut acheter une entreprise et vous cherchez une banque d'occasions d'affaires? Consultez la banque du marché virtuel ou déposez-y des offres et des demandes au nom de vos clients en toute confidentialité. Vous êtes de plus en plus occupé et vous cherchez un responsable de dossiers qui assumera une partie du travail? Le Centre d'emploi virtuel d'@BACUSTM apparie automatiquement votre offre d'emploi avec les CV qu'il a en banque.

Revue internationale P.M.E., vol. 15, nºs 3-4, 2002 


\section{Formation virtuelle}

Ce module diffuse des cours d'une durée de 3 ou de 7 heures ou des capsules de formation de 60 minutes portant sur des sujets pointus. Le cours répondra-t-il à vos besoins? Avant de le commander, lisez sa fiche technique. Pas encore convaincu? Testez vos connaissances avant de commander. Les cours sont diffusés dans Internet ou sur cédérom. Vous suivez le cours qui vous plaît, chez vous, au moment qui vous convient. Vous pourrez poser des questions à un animateur par courrier électronique. Aussi, un forum vous permettra d'échanger avec d'autres CA. Parce que votre temps est précieux, à chaque année, @BACUS ${ }^{\mathrm{TM}}$ prépare pour vous le rapport des activités de formation auxquelles vous avez participé et le courrielle à l'OCAQ.

\section{Pas du tout}

Certainement

Êtes-vous prêt à suivre des cours virtuels ?

Combien êtes-vous prêt à payer pour :

un cours de 7 heures?

un cours de 3 heures?

une capsule de 60 minutes?

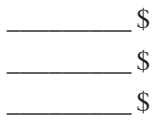

\section{$14 \quad$ Valeur d'@BACUS'T}

Regroupons tous ces modules dans un site.

Combien seriez-vous prêt à payer annuellement pour y avoir accès ?

\section{Classement des modules}

Voici la liste des modules composant @BACUS ${ }^{\mathrm{TM}}$. Classez-les par ordre d'importance $(1=\mathrm{le}$ plus intéressant; 13 = le moins intéressant).

1 Documentation officielle

2 Bases de connaissances et d'expertise

3 Cybermanuel de l'ICCA

4 Service de recherche en ligne

5 Applications transactionnelles

6 Certificats numériques (signatures électroniques)

7 Boutique virtuelle

8 Site Web de votre cabinet

9 Webdiffusion (push) de nouvelles

10 Échanges entre CyberCA

11 Répertoires virtuels

12 Marché virtuel

13 Formation virtuelle 


\section{Au sujet d'@BACUS ${ }^{\mathrm{TM}}$}

$1 \quad$ Aide à la navigation

En dépit des engins de recherche que vous trouverez dans @BACUSTM, vous aurez peut-être envie de confier votre recherche à un professionnel. Ce professionnel de recherche cherchera les renseignements dont vous avez besoin, moyennant rémunération.

\section{Pas du tout}

Certainement

Êtes-vous prêt à utiliser les services d'un professionnel de recherche?

$\begin{array}{lllllll}1 & 2 & 3 & 4 & 5 & 6 & 7\end{array}$

Quel taux horaire seriez-vous prêt à lui verser?

2 Qui devrait concevoir et exploiter @BACUS ${ }^{\mathrm{TM}}$ ?

( ) Un petit cabinet de CA.

( ) L'OCAQ

( ) Une université.

() Une entreprise qui œuvre dans le secteur des technologies de l'information.

() Un autre organisme (préciser):

$3 \quad$ Financement d'@BACUS ${ }^{\mathrm{TM}}$

( ) Accès à la carte.

( ) Abonnement mensuel donnant accès à certaines sections jumelé à un accès à la carte pour les modules plus coûteux.

( ) Abonnement mensuel avec accès à tous les modules.

( ) Cotisation spéciale établie en fonction du nombre de CA à l'emploi du cabinet.

\section{Bibliographie}

AICPA, CPA Vision Project (1998b). <http:/www.cpavision.org/> ; consulté les 25 août, 28 septembre, 12 et 18 octobre.

Amit, R. et C. Zотт (2000), Value Driver of e-Commerce Business Models, 41 pages, $<$ http://216.107.131.155/archive/papers/978.pdf>.

BERRY, E.L. (1978), «Quantitative criteria for evaluating overall performance of a government audit organization : an empirical study », The Government Accountants Journal, p. 43-53.

BERRY, E.L., G.B. HARwOOD et J.L. KATZ (1987), « Performance of auditing procedures by governmental auditors : some preliminary evidence », The Accounting Review, vol. LXII, $\mathrm{n}^{\mathrm{o}} 1$, janvier, p. 14-28.

BRACKNEY, K.S. et G.L. HELMS (1996), «A survey of attestation practices », Auditing, vol. $15, \mathrm{n}^{\mathrm{o}} 2$, automne, p. 85 .

BrILOFF, A. (1994), «Our profession "Jurassic Park" », The CPA Journal, vol. 64, août.

CARTIER, M. (2000), Les portails de $2^{e}$ génération : à quoi ressemblera la nouvelle économie, décembre, 55 pages.

Revue internationale P.M.E., vol. 15, $\mathrm{n}^{\text {os }} 3-4,2002$ 
DYER, J.H. (1997), «Effective interfirm collaboration : how firms minimize transaction costs and maximize transaction value », Strategic Management Journal, p. 535-556.

ELLiot, R.K. (1996), « Réinventer la vérification », CA Magazine, vol. 129, nº 6.

GRANOVETTER, M. (1985), «Economic action and social structure: the problem of embeddedness », American Journal of Sociology, vol. 91, p. 481-510.

HAGEL, J. et A.G. ARMSTRONG (1995), « Real profits from virtual communities », McKinsey Quarterly, no 3, p. 126-141.

Hagel, J. et A.G. Armstrong (1997), Net Gain : Expanding Markets Through Virtual Communities, Boston, MA, Harvard Business Press, 239 pages.

HELMS, G.L. et K.S. BRACKNEY (1998), «Attestation services : opportunity for practice growth », The CPA Journal, vol. 68, février.

HICKOK, R.S. (1982), «Shaping the future of accounting profession: a challenge and an opportunity », Journal of Accountancy, vol. 154, juillet.

Houle, K., S. NZAKImuena et M. VÉZINA (2002), Étude sur le taux de pénétration d'Internet par les cabinets comptables agréés établis au Québec, avril, 45 pages (non publié).

Jo KIM, A. (2000), Community Building on the Web, Peachpit Press, Berkeley, CA, 360 pages.

Karp, D., G. Stone, et W. Yoels (1977), Being Urban : A Social Psychological View of Life City, Lexington, MA, Health and Company.

LAFORTUNE, A. et J. MCNEIL (1993), «L'utilisation du micro-ordinateur en vérification au Canada : une recherche exploratoire. Comptabilité et nouvelles technologies », Actes du congrès de l'Association française de comptabilité, mai.

LAWRENCE, T.B. (1995), «Power and resources in an organisational community», Academy of Management Best Papers Proceedings, p. 251-255.

Lechner, U., K. Stanoevska-Slabeva et Y. Hua Tan (2002), "Communities in the digital economy », International Journal of Electronic Commerce, vol. 6, $\mathrm{n}^{\mathrm{0}} 3$, printemps, p. 5.

LEFEBVRE, É. et L.A. LEFEBVRE (2001), «Impact du commerce électronique sur la main d' œuvre», Technologies Polydev Inc., 40 pages.

LEGRAND, C.H. (1988), "The microcomputer as an audit tool and audit environment», EDPACS, mai.

MELAnÇON, B.C. (1998), «A path for the future», Journal of Accountancy, vol. 185, février.

O'Gorman, B. (1995), «Audit Automation», Journal of the Institute of Chartered Accountants in Ireland, octobre, p. 26-27.

Olson, W. (1980), «Our profession in a changing world», Journal of Accountancy, vol. $150, \mathrm{n}^{\circ} 5$.

OuEllet, P. et A. TRUdEAU (2001), Les affaires électroniques : tendances et enjeux pour la PME québécoise, Développement Économique Canada, février, 53 pages.

Revue internationale P.M.E., vol. 15, nos 3-4, 2002 
PENTLAND, B.T. (1989), «Use and productivity in personal computing : an empirical test», Proceedings of the Tenth International Conference on Information Systems, décembre, Boston, MA, p. 211-222.

Peat-Marwick (1987), Macintosh Benefits Study, Rapport interne, 24 pages.

PORTER, M. (2001), « Strategy and the Internet», Harvard Business Review, mars, p. 63-78.

POUSSART, B. (2001), Rapport d'enquête sur l'adoption du commerce électronique par les PME québécoises, Institut de la statistique du Québec, mars, 44 pages.

RothAERMEL, F. et S. SugIYAma (2001), « Virtual Internet communities and commercial success: individual and community-level theory grounded in the atypical case of TimeZone.com », Journal of Management, vol. 27, p. 297-312.

RoY, R. (2001), Guide sur les places d'affaires électroniques : tirez profit des portails, CEFRIO, 60 pages.

SHAPIRO, C. et H.R. VARIAN (1999), «The art of standard wars », California Management Review, hiver, vol. 41, Iss. 2, p. 8-32.

VAAST, E. (2001), «Les Intranets, occasions de renforcement et de transformation des communautés de pratiques: quatre études de cas », Actes de la $X^{e}$ conférence de l'Association internationale de management stratégique, juin, 26 pages.

VÉZINA, M. (1996), «L'impact des technologies de l'information sur la performance: résultats d'une enquête menée auprès des professionnels de la comptabilité », Systèmes d'information et management, vol. 1, automne.

Wolfe, C., C. BAin et W. MCPHETERS (1989), « Microcomputer productivity in the internal audit function : a case study », The Ohio CPA Journal, printemps, p. 12-16.

Revue internationale P.M.E., vol. 15, $\mathrm{n}^{\text {os }} 3-4,2002$ 